\title{
Long-term variability of the optical spectra of NGC 4151
}

\section{Evolution of the broad $\mathrm{H} \alpha$ and $\mathrm{H} \beta$ emission-line profiles}

\author{
A. I. Shapovalova ${ }^{1}$, L.Č. Popović2,3, A. N. Burenkov ${ }^{1}$, V. H. Chavushyan ${ }^{4}$, D. Ilić 3,5 , A. Kovačević ${ }^{3,5}$, \\ N. G. Bochkarev ${ }^{6}$, and J. León-Tavares ${ }^{4,7}$
}

${ }^{1}$ Special Astrophysical Observatory of the Russian AS, Nizhnij Arkhyz, Karachaevo-Cherkesia 369167, Russia e-mail: ashap@sao.ru

2 Astronomical Observatory, Volgina 7, 11160 Belgrade 74, Serbia

3 Isaac Newton Institute of Chile, Yugoslavia Branch

4 Instituto Nacional de Astrofísica, Óptica y Electrónica, Apartado Postal 51, CP 72000, Puebla, México

5 Department of Astronomy, Faculty of Mathematics, University of Belgrade, Studentski trg 16, 11000 Belgrade, Serbia

6 Sternberg Astronomical Institute, Moscow, Russia

7 Metsähovi Radio Observatory, Helsinki University of Technology TKK, Metsähovintie 114, 02540 Kylmälä, Finland

Received 9 April 2009 / Accepted 10 October 2009

ABSTRACT

\begin{abstract}
Aims. We present the results of a long-term monitoring (11 years, between 1996 and 2006) of $\mathrm{H} \alpha$ and $\mathrm{H} \beta$ line variations of the active galactic nucleus of NGC 4151.

Methods. High quality spectra $(S / N>50$ and $R \approx 8 \AA$ ) of $\mathrm{H} \alpha$ and $\mathrm{H} \beta$ were investigated. During monitoring period, we analyzed line profile variations. Comparing the line profiles of $\mathrm{H} \alpha$ and $\mathrm{H} \beta$, we studied different details (bumps, absorbtion features) in the line profiles. The variations in the different $\mathrm{H} \alpha$ and $\mathrm{H} \beta$ line profile segments were investigated. We also analyzed the Balmer decrement for entire lines and for line segments.

Results. We found that the line profiles varied strongly during the monitoring period, and exhibited blue and red asymmetries. This is indicative of a complex BLR geometry inside NGC 4151 with, at least, three kinematically distinct regions: one that contributes to the blue line wing, one to the line core and one to the red line wing. The variation may be caused by an accelerating outflow originating very close to the black hole, where the red part may come from a region closer to the black hole than the blue part, which originates in the region with the highest outflow velocities.

Conclusions. Taking into account that the BLR of NGC 4151 has a complex geometry (probably affected by an outflow) and that a portion of the broad line emission does not seem to be produced entirely by photoionization, one may ask whether the study of the BLR using reverberation mapping would be worthwhile for this galaxy.
\end{abstract}

Key words. galaxies: active - galaxies: individual: NGC 4151

\section{Introduction}

In spite of many papers being devoted to the physical properties (physics and geometry, see e.g., Sulentic et al. 2000) of the broad line region (BLR) in active galactic nuclei (AGN), the true nature of the BLR is not well known. The broad emission lines, and their shapes and intensities can provide much information about the BLR geometry and physics. In the first instance, the change in the line profiles and intensities could be used to investigate the BLR nature. It is often assumed that variations in line profiles on long timescales are caused by the dynamic evolution of the BLR gas, and on short timescales by reverberation effects (Sergeev et al. 2001). In a number of papers, it has been shown that individual segments in the line profiles change independently, on both long and short timescales (e.g., Wanders \& Peterson 1996; Kollatschny \& Dietrich 1997; Newman et al. 1997; Sergeev et al. 1999). The broad line shapes may also provide information about the kinematics and structure of the BLR (see Popović et al. 2004).

One of the most famous and well studied Seyfert galaxies is NGC 4151 (see e.g. Ulrich 2000; Sergeev et al. 2001; Lyuty 2005, Shapovalova et al. 2008 - Paper I, and reference therein).
This galaxy, and its nucleus, has been studied extensively at all wavelengths. The reverberation investigation indicates a small BLR size in the center of NGC 4151 (see e.g. Peterson \& Cota 1988: $6 \pm 4$ 1.d.; Clavel et al. 1990: $4 \pm 3$ 1.d.; Maoz et al. 1991: $9 \pm 2$ 1.d.; Bentz et al. 2006: 6.6 $6_{+1.1}^{-0.8}$ 1.d.).

Spectra of NGC 4151 show a P Cygni Balmer and He I absorption with an outflow velocity ranging between $-500 \mathrm{~km} \mathrm{~s}^{-1}$ and $-2000 \mathrm{~km} \mathrm{~s}^{-1}$, which varies with the nuclear flux (Anderson \& Kraft 1969; Anderson 1974; Sergeev et al. 2001; Hutchings et al. 2002). This material is moving outward along the line of sight, and may be anywhere beyond $\sim 15$ light-days (Ulrich \& Horne 1996). An outflow is also seen in higher velocity emission-line clouds close to the nucleus (Hutchings et al. 1999), such as multiply shifted absorption lines in CIV and other UV resonance lines (Weymann et al. 1997; Crenshaw et al. 2000), while warm absorbers are detected in X-ray data (e.g., Schurch \& Warwick 2002).

Some authors assumed that variable absorption is responsible, at least partially, for the observed continuum variability of AGN (Collin-Souffrin et al. 1996; Boller et al. 1997; Brandt et al. 1999; Abrassart \& Czerny 2000; Risaliti et al. 2002). Czerny et al. (2003) considered that most variations are 
intrinsic to the source, although variable absorption cannot be quite excluded.

The nucleus of NGC 4151 also emits in the radio range. The radio image detects a $0.2 \mathrm{pc}$ two-sided base to the wellknown arcsecond radio jet (Ulvestad et al. 2005). The apparent speeds of the jet components relative to the radio AGN are less than $0.050 \mathrm{c}$ and less than $0.028 \mathrm{c}$, at nuclear distances of 0.16 and $6.8 \mathrm{pc}$, respectively. These represent the lowest speed limits yet found in a Seyfert galaxy and are indicative of non-relativistic jet motions, possibly due to thermal plasma, on a scale only an order of magnitude larger than the BLR (Ulvestad et al. 2005).

The observed evolution of the line profiles of the Balmer lines of NGC 4151 was studied by Sergeev et al. (2001) in 1988-1998 and was modeled well within the framework of the two-component model, where two variable components with fixed line profiles (double-peaked and single-peaked) were used.

Although the AGN of NGC 4151 has been well observed and discussed, there are several outstanding questions about the BLR kinematics (disk, jets, or more complex BLR) and dimensions of the innermost region. On the other hand, as mentioned above, multiwavelength observations indicate that both an accretion disk (with a high inclination) and outflow emission (absorption) are present. Consequently, further investigations of the NGC 4151 nucleus are needed to constrain the kinematics, dimensions, and geometry of its BLR.

This work proceeds Paper I and aims to study the variations in both the integrated profiles of the broad emission lines and segments along the line profiles, during the (11-year) period of monitoring of NGC 4151.

The paper is organized as follows: in Sect. 2, observations and data reduction are presented. In Sect. 3, we study the averaged spectral line profiles (over years, months, and Periods I-III, see Paper I) of $\mathrm{H} \alpha$ and $\mathrm{H} \beta$, their line asymmetries and their $F W H M$ variations, light curves of different line segments, and the line-segment to line-segment flux and continuumline-segment relations. In Sect. 4, we analyze the Balmer decrements. The results are discussed in Sect. 6 and in Sect. 7 we outline our conclusions.

\section{Observations and data reduction}

Optical spectra of NGC 4151 were taken with the 6-m and 1-m telescopes of SAO, Russia (1996-2006), with the 2.1-m telescope of the Guillermo Haro Astrophysical Observatory (GHAO) at Cananea, Sonora, México (1998-2006), and with the 2.1-m telescope of the Observatorio Astronómico Nacinal at San Pedro Martir (OAN-SMP), Baja California, México (2005-2006). They were obtained with a long-slit spectrograph equipped with CCDs. The typical wavelength range was 4000-7500 $\AA$, the spectral resolution was $R=5-15 \AA$, and the $S / N$ ratio was $>50$ in the continuum near $\mathrm{H} \alpha$ and $\mathrm{H} \beta$. In total, 180 blue and 137 red spectra were taken during 220 nights.

Spectrophotometric standard stars were observed each night. The spectrophotometric data reduction was carried out with either software developed at the SAO RAS by Vlasyuk (1993), or IRAF for the spectra observed in México. The image reduction process included bias subtraction, flat-field corrections, cosmic ray removal, 2D wavelength linearization, sky spectrum subtraction, addition of the spectra for every night, and relative flux calibration based on standard star observations. Spectra were scaled to the constant flux F([O III $] \lambda$ 5007). More details about observations and data reduction are given in Paper I and are not repeated here.
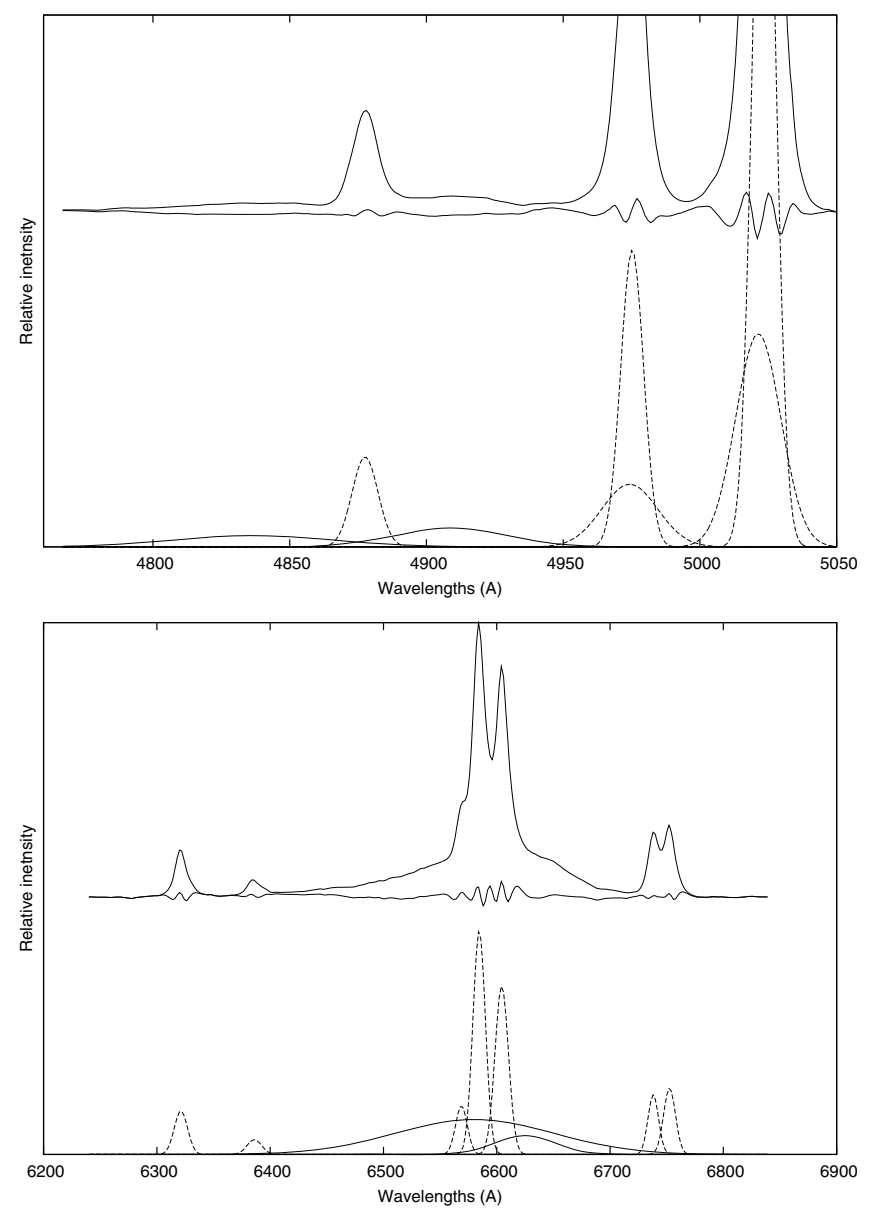

Fig. 1. The decomposition of $\mathrm{H} \beta$ (upper panel) and $\mathrm{H} \alpha$ (bottom panel) into Gaussians (below) to construct narrow line templates. The dashed Gaussians correspond to the narrow components taken for the narrow line templates, while the solid ones correspond to the broad components (down). The observed spectra and rms (up) are denoted with solid line.

The observed fluxes of the emission lines were corrected for position angle (PA), seeing, and aperture effects (see Paper I). The mean error (uncertainty) in our integral flux determinations for $\mathrm{H} \alpha$ and $\mathrm{H} \beta$ and for the continuum is $<3 \%$. To study the broad components of emission lines and the main BLR characteristics, we removed from the spectra the narrow components of these lines and the forbidden lines. For this purpose, we construct spectral templates using the blue and red spectra in the minimum activity state (May 12, 2005). Both the broad and narrow components of $\mathrm{H} \beta$ and $\mathrm{H} \alpha$, were fitted with Gaussians (see Fig. 1). The template spectrum contains the following lines: for $\mathrm{H} \beta$, the narrow component of $\mathrm{H} \beta$ and $[\mathrm{O}$ III $] \lambda \lambda 4959,5007$; and for $\mathrm{H} \alpha$ the narrow component of $\mathrm{H} \alpha,[\mathrm{N} \mathrm{II}] \lambda \lambda 6548,6584$, [OI] $\lambda \lambda 6300,6364,[\mathrm{SII}] \lambda \lambda 6717,6731$. We then scaled the blue and red spectra according to our scaling scheme (see the appendix of Shapovalova et al. 2004), using the template spectrum as reference. The template spectrum and any observed spectrum are thus matched in wavelength, brought to the same resolution, and the template spectrum is then subtracted from the observed one. More details can be found in Paper I and Shapovalova et al. (2004).

\section{Line profile variations}

To investigate the broad line profile variations, we use the most intense broad lines in the observed spectral range, i.e., $\mathrm{H} \alpha$ and 
$\mathrm{H} \beta$, only from spectra of spectral resolution $\sim 8 \AA$. In Paper I, we defined 3 characteristic time periods (I: 1996-1999, II: 2000-2001, and III: 2002-2006) during which the line profiles of these lines were similar. Average values and rms profiles of both lines were obtained during these periods.

Here we recall some of the most important results of Paper I. In the first period (I, 1996-1999, JD = 2450 094.5-2 451 515.6), when the lines were most intense, a highly variable blue component was observed, which exhibited two peaks or shoulders at $\sim-4000 \mathrm{~km} \mathrm{~s}^{-1}$ and $\sim-2000 \mathrm{~km} \mathrm{~s}^{-1}$ in the rms $\mathrm{H} \alpha$ profiles and, to a lesser degree, in $\mathrm{H} \beta^{1}$. In the second period (II, 2000-2001, JD = 2451 552.6-2 452 238.0), the broad lines were much fainter, the feature at $\sim-4000 \mathrm{~km} \mathrm{~s}^{-1}$ was disappearing from the blue part of the rms profiles of both lines, and only the shoulder at $\sim-2000 \mathrm{~km} \mathrm{~s}^{-1}$ was present. A faint shoulder at $\sim 3500 \mathrm{~km} \mathrm{~s}^{-1}$ was present in the red part of rms line profiles (see Fig. 6 in Paper I). In the third period (III, 2002-2006, JD = 2452 299.4-2 453 846.4), a red feature (bump, shoulder) at $\sim 2500 \mathrm{~km} \mathrm{~s}^{-1}$ was clearly seen in the red part of both the mean and the rms line profiles (see Fig. 7 in Paper I). In this paper, we study the variations in the broad line profiles in more detail.

\subsection{Monthly- and yearly-averaged profiles of the broad $\mathrm{H} \alpha$ and $H \beta$ lines}

A rapid inspection of spectra shows that the broad line profiles vary negligibly within a one-month interval. On the other hand, in this time-interval, a slight variation in the broad line flux is evident (usually around $\sim 5-10 \%$, except in some cases when it is up to $30 \%$ ). Therefore we constructed the monthly-averaged line profiles (see Fig. 2) of $\mathrm{H} \alpha$ and $\mathrm{H} \beta$.

The broad $\mathrm{H} \alpha$ and $\mathrm{H} \beta$ line profiles did not vary significantly within a one-year period (or even during several years), while the broad line fluxes sometimes varied by factors $\sim 2-2.5$ even within one year. The smallest flux variations (factors $\sim 1.1-1.3$ ) were observed in 1996-1998 (during the line flux maximum). The largest line flux variations were observed in 2000-2001 and 2005 (factors $\sim 1.7-2.5$ ), during the minimum of activity.

As mentioned in Paper I, specific line profiles are observed during the three periods, but a more detailed inspection of the line profiles shows that slight changes can also be seen between the yearly-averaged profiles (see Fig. 3). We note here that in the central part of the $\mathrm{H} \alpha$ profiles one can often see considerable residuals (e.g., in 2001 in the form of peaks) because of poor subtraction of the bright narrow components of $\mathrm{H} \alpha$ and [N II] $\lambda \lambda 6548,6584$ (at $V_{r} \sim-680 \mathrm{~km} \mathrm{~s}^{-1}$ and $\sim 960 \mathrm{~km} \mathrm{~s}^{-1}$ ). Therefore, we cannot conclude anything about the presence of absorption in the central part of $\mathrm{H} \alpha$. But, in the $\mathrm{H} \beta$ profiles (in the central part, at $V_{r} \sim-430 \mathrm{~km} \mathrm{~s}^{-1} ; \sim-370 \mathrm{~km} \mathrm{~s}^{-1}$ ) an absorption, especially strong from June 1999 to the end of 2000 (see Fig. 2), was detected. We note here that Hutchings et al. (2002) also found absorbtion at $V_{r}(-2000-0) \mathrm{km} \mathrm{s}^{-1}$ in the $\mathrm{H} \beta$ line.

We now note some noticeable features in monthly-averaged line profiles:

1. in 1996-2001, a blue peak (bump) in $\mathrm{H} \beta$ and a shoulder in $\mathrm{H} \alpha$ were clearly seen at $\sim-2000 \mathrm{~km} \mathrm{~s}^{-1}$ (Fig. 2). However, in 2002-2004, the blue wing of both lines became steeper than the red one and it did not contain any noticeable features;

\footnotetext{
${ }^{1}$ Here and after in the text the radial velocities are given with respect to the corresponding narrow components of $\mathrm{H} \alpha$ or $\mathrm{H} \beta$, i.e. it is accepted that $V_{r}=0$ for the narrow components of $\mathrm{H} \alpha$ and $\mathrm{H} \beta$.
}

2. in 2005 (May-June), when the nucleus of NGC 4151 was experiencing its minimum of activity, the line profiles had a double-peak structure with two distinct peaks (bumps) at radial velocities of $(-2586 ;+2027) \mathrm{km} \mathrm{s}^{-1}$ in $\mathrm{H} \beta$ and $(-1306 ;+2339) \mathrm{km} \mathrm{s}^{-1}$ in $\mathrm{H} \alpha$ (see Fig. 2; 2005_05 and 2005_06). In principle, the two-peak structure in the $\mathrm{H} \beta$ profiles is also seen in the spectra of 1999-2001: at $V_{r} \sim$ $-1500 \mathrm{~km} \mathrm{~s}^{-1}$ the blue and $V_{r} \sim 500 \mathrm{~km} \mathrm{~s}^{-1}$ the red peak. However, in this case the blue peak may be caused by a broad absorption line at the radial velocity $\left(\sim-400 \mathrm{~km} \mathrm{~s}^{-1}\right)$;

3 . in 2006 , the line profiles changed dramatically - the blue wing became flatter than in previous periods, while the red wing was very steep without any feature at $V_{r}>$ $2300 \mathrm{~km} \mathrm{~s}^{-1}$;

4. In 2002, a distinct peak (bump) appeared in the red wing of the $\mathrm{H} \alpha$ and $\mathrm{H} \beta$ lines at the radial velocity $V_{r} \sim 3000 \mathrm{~km} \mathrm{~s}^{-1}$. The radial velocity of the red peak decreases: in 2002-2003, it corresponds to $\sim 3100 \mathrm{~km} \mathrm{~s}^{-1}$ and in 2006 to $\sim 2100 \mathrm{~km} \mathrm{~s}^{-1}$. This effect is clearly seen in Fig. 4, especially in the $\mathrm{H} \alpha$ line profile. Table 1 provides the obtained radial velocities of red peak measured in the $\mathrm{H} \alpha$ and $\mathrm{H} \beta$ profiles for which the peak was clearly seen. Radial velocities of the $\mathrm{H} \alpha$ and $\mathrm{H} \beta$ red peak, measured over the same period, are similar (the differences are within the errorbars of measurements). The mean radial velocity of the red peak decreased by $\sim 1000 \mathrm{~km} \mathrm{~s}^{-1}$ from 2002 to 2006 (Table 1). It is unclear whether the red peak is shifting along the line profile or it disappears and again appears as a new red peak at another velocity.

\subsection{The absorption and emission features in $\mathrm{H} \alpha$ and $\mathrm{H} \beta$ line profiles}

As mentioned above (Sect. 3.1), the $\mathrm{H} \alpha$ and $\mathrm{H} \beta$ line profiles have both absorption and emission features, and distinguishing between these features is very difficult. The question is are the "bumps" an intrinsic property of the broad $\mathrm{H} \alpha$ and $\mathrm{H} \beta$ lines or do they just strengthen the absorption features? Furthermore, an open question what is the origin of these features, i.e., is there an intrinsic mechanism in the BLR that creates these "bumps"? We should mention here that in the central part of both lines, the residuals of the narrow components can affect these relatively weak absorption/emission features.

To study it, the residuals between some $\mathrm{H} \beta$ broad lines with prominent (noticeable) bumps from in particular two successive months were obtained. An example of these residuals are presented in Fig. 5. The noticeable residual bumps (without an absorption-like feature) at $V_{r}$ from $(-2000) \mathrm{km} \mathrm{s}^{-1}$ to $(-1000) \mathrm{km} \mathrm{s}^{-1}$ in $1999-2001$, and at $V_{r}$ from $3500 \mathrm{~km} \mathrm{~s}^{-1}$ to $2500 \mathrm{~km} \mathrm{~s}^{-1}$ in 2002-2006 are seen well. It seems that the absorption changes slowly and during several next months remains constant. Therefore, this absorption disappears in profile residuals (see Fig. 5). Consequently, it seems that emission bumps observed in the $\mathrm{H} \beta$ and $\mathrm{H} \alpha$ line profiles (seen in the residuals in Fig. 5) are mostly an intrinsic property of the broad emissionline profile.

The strong absorption features are also present in 1996-2001 H $\beta$ spectra. In this period, we observed the dip and broad absorption at radial velocity that changes from $\sim-1000 \mathrm{~km} \mathrm{~s}^{-1}$ in $1996-1998$ to $\sim-400 \mathrm{~km} \mathrm{~s}^{-1}$ in $1999-2000$ (see Fig. 2). This velocity corresponds to the minimum of the absorption band, but its blue edge extends to higher velocities $\left(\sim-1800 \mathrm{~km} \mathrm{~s}^{-1}\right.$ in 1998 and $\sim-1170$ in 1999-2000). Figure 6 shows some observed individual spectra and their broad 

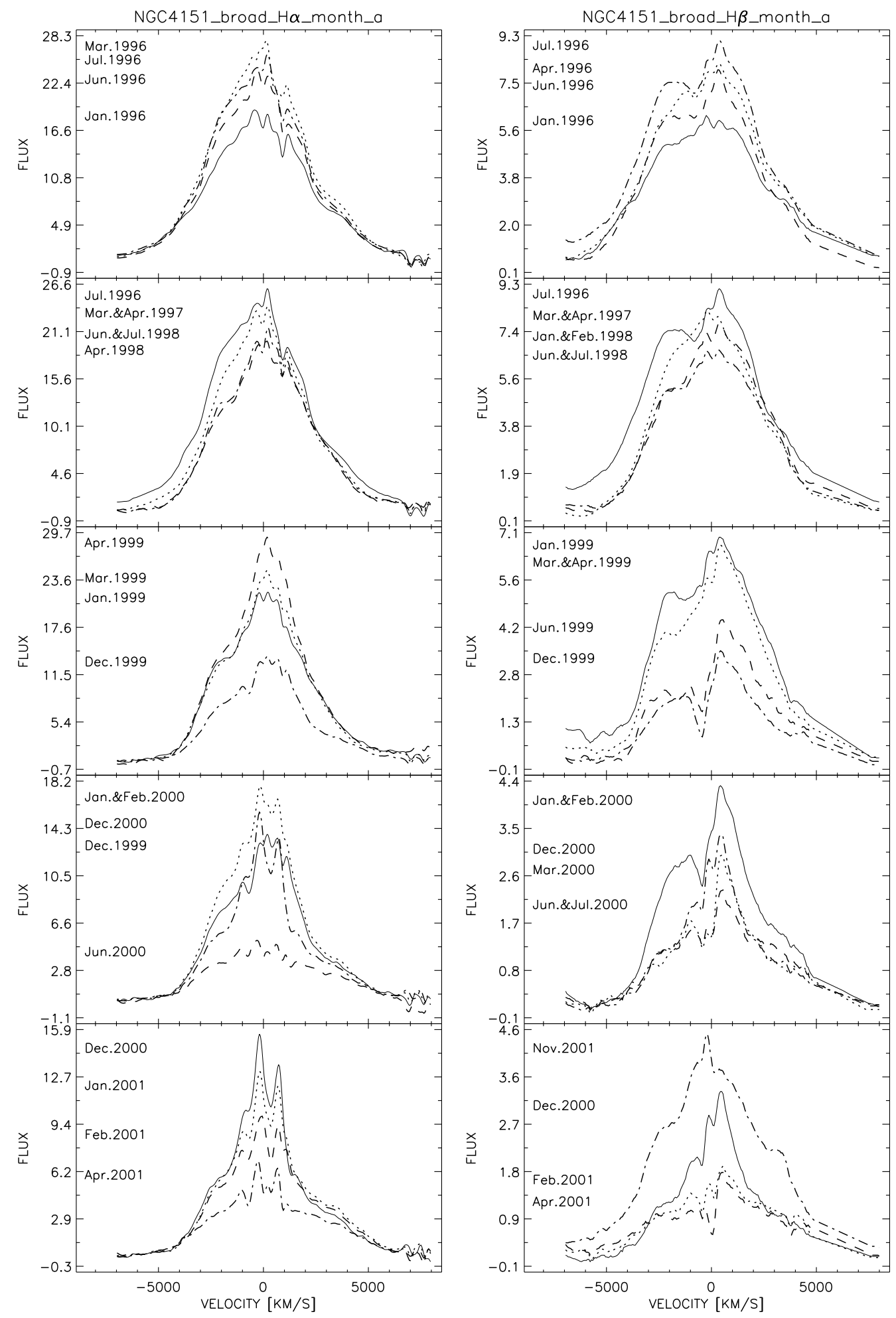

Fig. 2. The monthly-averaged profiles of the $\mathrm{H} \alpha$ and $\mathrm{H} \beta$ broad emission lines in the period 1996-2006. The abscissae shows the radial velocities relative to the narrow component of $\mathrm{H} \alpha$ or $\mathrm{H} \beta$. The ordinate shows the flux in units of $10^{-14} \mathrm{erg} \mathrm{cm}^{-2} \mathrm{~s}^{-1} \AA^{-1}$.

component where the blue absorption is well resolved. The blueshifted absorption probably originates from outflowing material.

It is interesting to note that the higher radial velocity $\left(\sim-1000 \mathrm{~km} \mathrm{~s}^{-1}\right.$, observed in 1996-1998) appears at a higher continuum flux level, while the smaller velocity $\left(\sim-400 \mathrm{~km} \mathrm{~s}^{-1}\right)$ was measured when the continuum flux had decreased 3-6 times, i.e., we confirm the results reported by
Hutchings et al. (2002), who found the same trend that the outflow velocity increases with the continuum flux.

\subsection{Asymmetry of the broad $\mathrm{H} \alpha$ and $\mathrm{H} \beta$ line profiles}

We measured the full width at half maximum (FWHM) of the broad lines from their monthly-averaged profiles and determined 


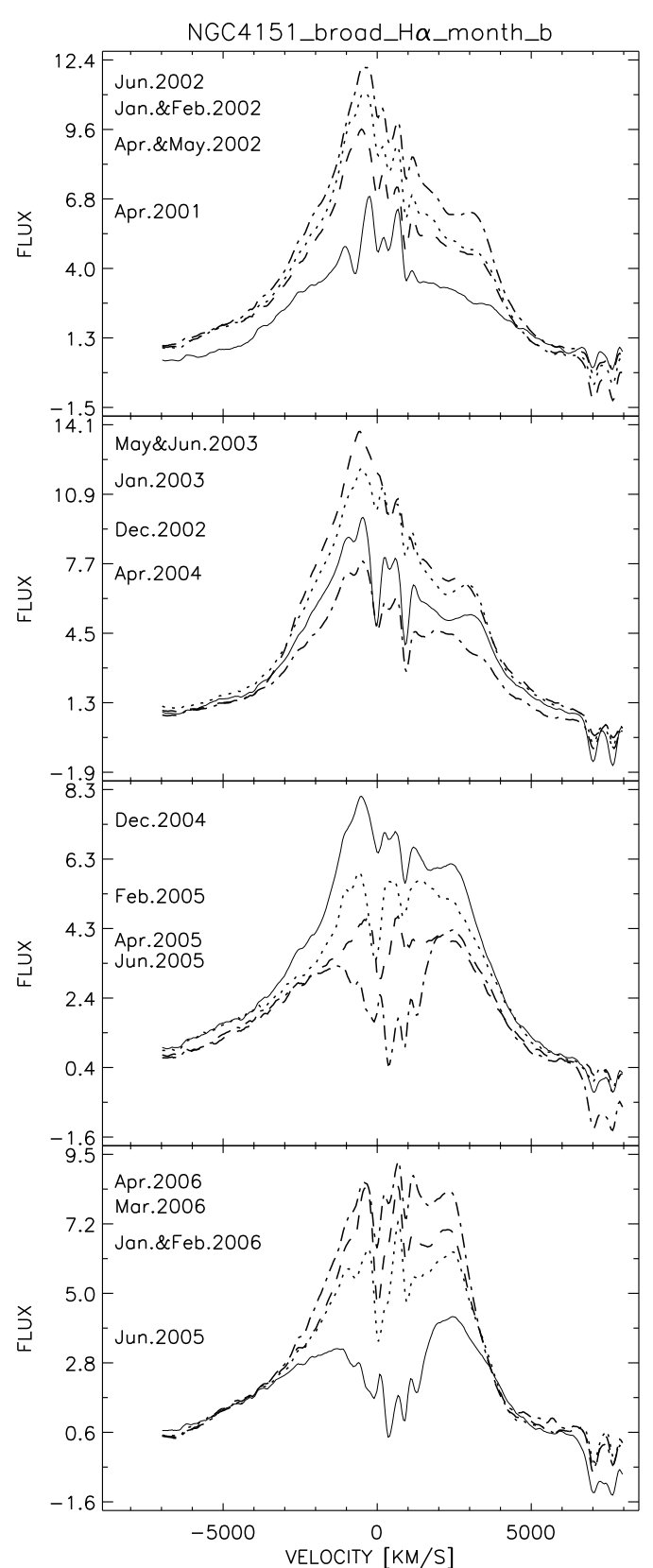

Fig. 2. continued.

the asymmetry $(A)$ as a ratio of the red to blue parts of $F W H M$, i.e., $A=W_{\text {red }} / W_{\text {blue }}$, where $W_{\text {red }}$ and $W_{\text {blue }}$ are the red and blue half-widths at maximal intensity (see Fig. 7) with respect to the position of the narrow component of $\mathrm{H} \alpha$ and $\mathrm{H} \beta$. As we mentioned above, there are residuals at the center of the $\mathrm{H} \alpha$ and $\mathrm{H} \beta$ lines due to residuals from the subtraction of narrow components, which can affect the $F W H M$ and $A$ measurements. Therefore, we first smoothed the line profiles, to avoid artificial peaks from the residuals (see Fig. 7), before measuring the FWHM and asymmetry. The two independent measurements of FWHM and A were performed. We determined the averaged continuum and its dispersion for each month and the averaged Julian date from the spectra, which were used to construct the monthly-averaged profiles. The measurements of the FWHM, asymmetry, and continuum during the entire period of monitoring (1996-2006) are presented in Table 2 and in Fig. 8. In

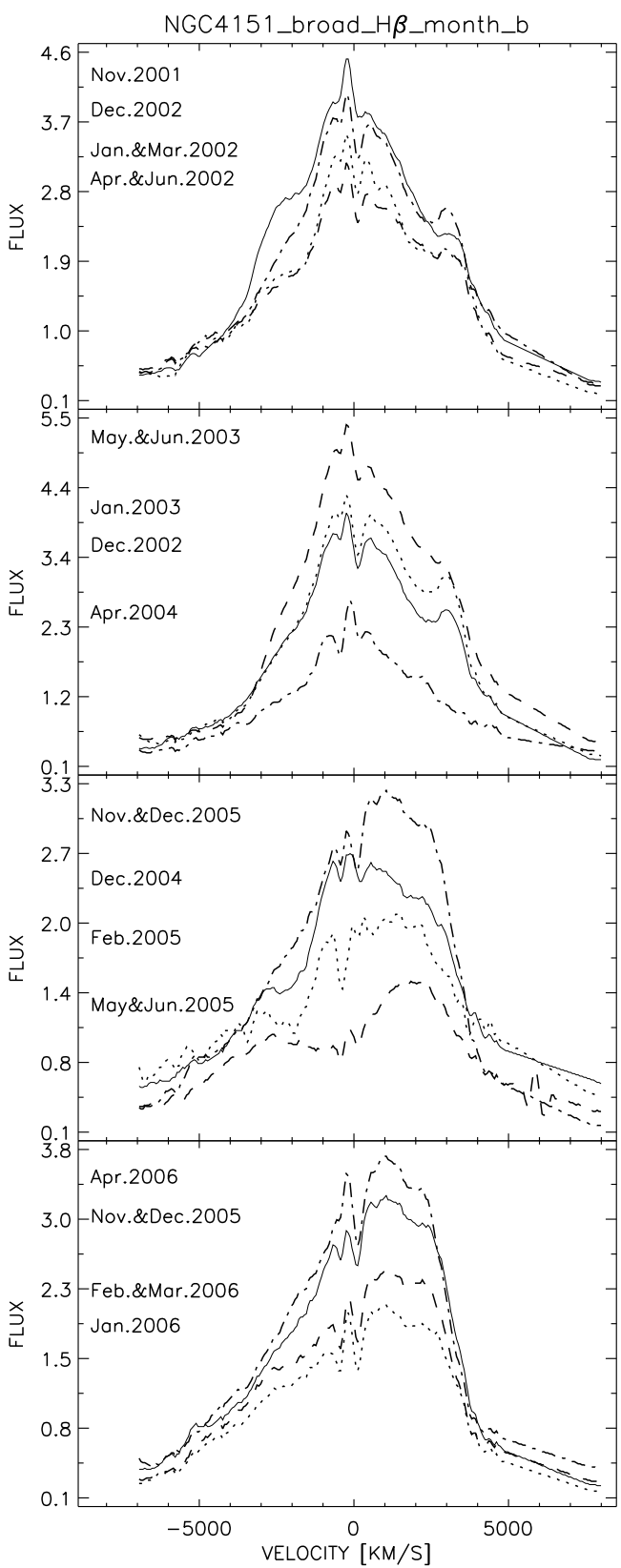

Table 2, we give average values of the FWHM and A from two independent measurements and their dispersions.

The $F W H M$ of $\mathrm{H} \beta$ was almost always larger than that of $\mathrm{H} \alpha$ (see upper panel of Fig. 8). The asymmetry of both lines (middle panel of Fig. 8) gradually increased between 1996 and 2006 and it slightly anticorrelates with the variations in the continuum (bottom panel in Fig. 8). The largest values of the FWHM and an outstanding red asymmetry of both lines $(A>1.2)$ was observed in 2002-2006. We calculated average values of the $F W H M$ and A each year, as well as for the whole monitoring period (they are given in Table 2). FWHMs and asymmetries obtained in this work from measurements of the monthly-average profiles are similar to the results given in Paper I and difference between them are within the error bars. As one can see from Table 2, the $F W H M$ of both lines varied rather considerably from year to year $\left(\triangle F W H M \sim 500-1500 \mathrm{~km} \mathrm{~s}^{-1}\right)$. These lines were at their narrowest in 2000-2001 (FWHM $\left.\sim 4000-5000 \mathrm{~km} \mathrm{~s}^{-1}\right)$ 

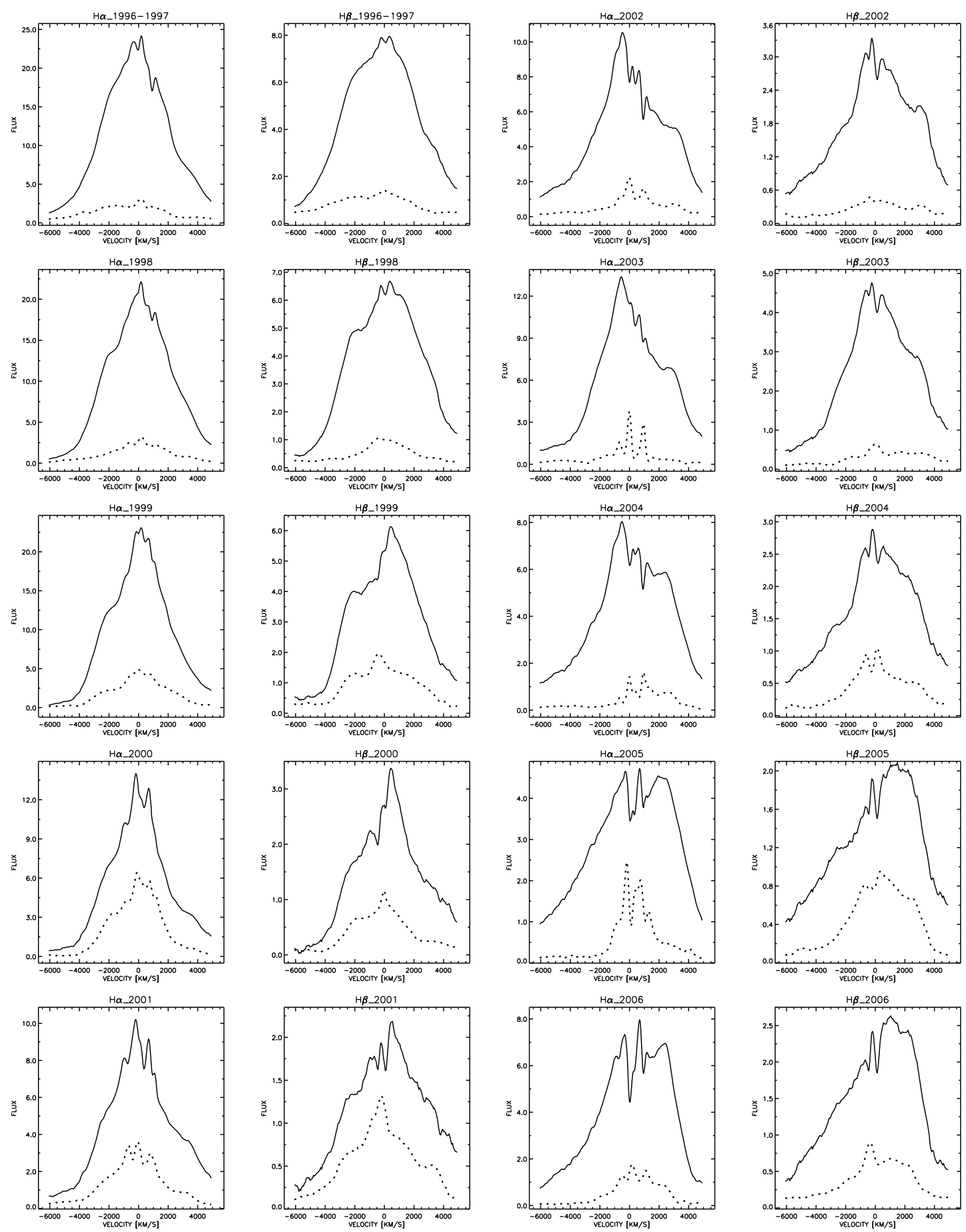

Fig. 3. The yearly-averaged profiles (solid line) and their rms (dashed line) of the $\mathrm{H} \alpha$ and $\mathrm{H} \beta$ broad emission lines in 1996-2006. The abscissae (OX) shows the radial velocities relative to the narrow component of the $\mathrm{H} \alpha$ or $\mathrm{H} \beta$ line. The ordinate (OY) shows the flux in units of $10^{-14} \mathrm{erg} \mathrm{cm}^{-2} \mathrm{~s}^{-1} \AA^{-1}$.

and their broadest in $2005\left(\sim 7000 \mathrm{~km} \mathrm{~s}^{-1}\right)$ (see Table 2). At the same time, the $\mathrm{H} \beta F W H M$ was always broader than $\mathrm{H} \alpha$ by on average $\sim 1000 \mathrm{~km} \mathrm{~s}^{-1}$. The asymmetry varied in different ways: in 1996-1997, the blue asymmetry was observed in $\mathrm{H} \beta$ $(A \sim 0.85)$ when the continuum flux was at its maximum; in 1998-2000, $\mathrm{H} \beta$ was almost symmetric, and from 2001 to 2006 the red asymmetry appeared $(A>1.2)$. In 1996-2001, a blue symmetry $(A \sim 0.8)$ was observed in $\mathrm{H} \alpha$, and in 2002-2006 a red asymmetry $(A>1.2)$ was observed.

We also tried to identify correlations of both the $F W H M$ and A with the continuum flux, and found that the FWHM practically does not correlate with the continuum level $(r \sim$ $0.0 ;-0.1)$. In the case of the asymmetry A, there is an indication of anticorrelation, but it should also be interpreted with caution since there is a large scatter of points in the A versus continuum flux plane, especially in the case of low continuum fluxes $F_{\mathrm{c}}<5.3 \times 10^{-14} \mathrm{erg} \mathrm{cm}^{-2} \mathrm{~s}^{-1} \AA^{-1}$ when the measured asymmetry reaches its highest values. We note that a photoinoization model predicts that the Balmer lines should be broader in the lower continuum states and narrower in the higher continuum states (see Korista \& Goad 2004), since, because of the greater response of the line cores, one can expect that Balmer lines 
Table 1. The peak shifts in the red wing of the $\mathrm{H} \alpha$ and $\mathrm{H} \beta$ lines.

\begin{tabular}{|c|c|c|c|}
\hline $\begin{array}{l}\mathrm{H} \alpha \\
\text { year_month }\end{array}$ & $\begin{array}{l}V_{r}(\mathrm{H} \alpha) \\
\mathrm{km} \mathrm{s}^{-1}\end{array}$ & $\begin{array}{c}\mathrm{H} \beta \\
\text { year_month }\end{array}$ & $\begin{array}{l}V_{r}(\mathrm{H} \beta) \\
\mathrm{km} \mathrm{s}^{-1}\end{array}$ \\
\hline & & 2001b_11 & 3257 \\
\hline 2002_1_3 & 3068 & 2002_1_3 & 3257 \\
\hline 2002_4_5 & 3114 & 2002_4_6 & 3196 \\
\hline 2002 r_o6 & 3068 & & \\
\hline 2002r_12 & 2977 & 2002b_12 & 3073 \\
\hline mean 2002 & $3057 \pm 57$ & mean 2001_11 + 2002 & $3197 \pm 94$ \\
\hline 2003r_01 & 2886 & 2003b_01 & 3073 \\
\hline 2003r_05 & 2613 & 2003b_03 & 3012 \\
\hline 2003r_06 & 2613 & 2003_5_6 & 2889 \\
\hline mean 2003 & $2704 \pm 158$ & mean 2003 & $2991 \pm 94$ \\
\hline 2004r_12 & 2339 & 2004b_12 & 2273 \\
\hline 2005r_02 & 2294 & 2005_1_2 & 2027 \\
\hline 2005r_04 & 2294 & 2005_5_6 & 2027 \\
\hline 2005r_06 & 2339 & 2005_11_12 & 2338 \\
\hline \multirow[t]{2}{*}{ 2006_1_2 } & 2430 & 2006b_01 & 2335 \\
\hline & & 2006b_02 & 2335 \\
\hline 2006r_03 & 2202 & 2006b_03 & 2150 \\
\hline 2006r_04 & 2294 & 2006b_04 & 2027 \\
\hline mean 2004-2006 & $2313 \pm 69$ & mean 2004-2006 & $2189 \pm 147$ \\
\hline
\end{tabular}

Columns: 1 - year and month for $\mathrm{H} \alpha$ (e.g., 2002_1_3 = 2002, January and March); $2-V_{r}(\mathrm{H} \alpha), \mathrm{H} \alpha$ peak velocity in the red wing $\left(\mathrm{km} \mathrm{s}^{-1}\right) ; 3$ - year and month for $\mathrm{H} \beta ; 4-V_{r}(\mathrm{H} \beta), \mathrm{H} \beta$ peak velocity in the red wing $\left(\mathrm{km} \mathrm{s}^{-1}\right)$. The line after each year (or interval of years) gives the mean $V_{\mathrm{r}}(\mathrm{H} \alpha)$ and $V_{r}(\mathrm{H} \beta)$ and their standard deviations. The last line gives the maximal shift of the red peak $\left(\Delta V_{r}\right)$ from 2002 to 2006.
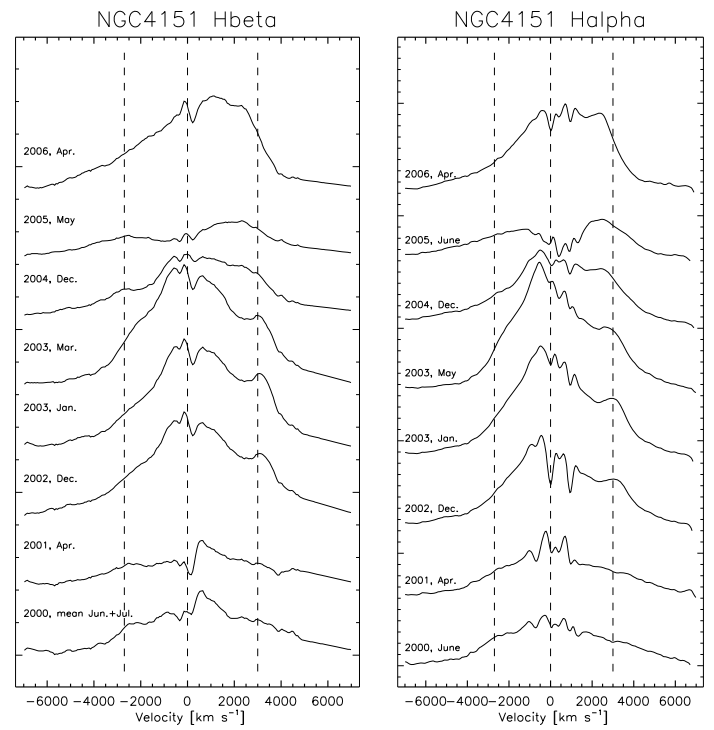

Fig. 4. Some examples of monthly-averaged profiles of the $\mathrm{H} \alpha$ and $\mathrm{H} \beta$ broad emission lines from 2000 to 2006. The abscissae shows the radial velocities relative to the narrow component of $\mathrm{H} \alpha$ or $\mathrm{H} \beta$. The vertical dashed lines correspond to radial velocities: $-2600 \mathrm{~km} \mathrm{~s}^{-1}, 0 \mathrm{~km} \mathrm{~s}^{-1}$, and $3000 \mathrm{~km} \mathrm{~s}^{-1}$. The profiles are shifted vertically by a constant value.

become narrower in higher continuum states. As one can see from Table 2, there is no trend for $F W H M$ to be significantly narrower in the high continuum state.

\subsection{Light curves of different line segments}

In Paper I, we obtained light curves of the integrated flux in lines and continuum. To study the BLR in more detail, we start in this paper by assuming that a portion of the broad line profile can respond to variations in the continuum in different ways. Therefore, we divided the line profiles into 11 identical profile segments, each of width $\sim 1000 \mathrm{~km} \mathrm{~s}^{-1}$ (see Table 3).

The observational uncertainties were determined for each segment of the $\mathrm{H} \beta$ and $\mathrm{H} \alpha$ light curves. In evaluating the uncertainties, we account for errors due to: the position angle correction, seeing correction procedure, and aperture correction. The methods for evaluating these uncertainties (errorbars) are given in Paper I. The effect of the subtraction of the template spectrum (or the narrow components) was studied by comparing the flux of pairs of spectra obtained in the time interval from 0 to 2 days. In Table 4, we presented the yearly-averaged uncertainties (in percent) for each segment of $\mathrm{H} \alpha$ and $\mathrm{H} \beta$ and mean values for all segments and corresponding mean-year flux. To determine the errorbars, we used 44 pairs of $\mathrm{H} \alpha$ and 68 pairs of $\mathrm{H} \beta$. As one can see from Table 4 , for the far wings (segments \pm 5 ) the errorbars are greater $(\sim 10 \%)$ in $\mathrm{H} \beta$ than in $\mathrm{H} \alpha(\sim 6 \%)$. But when comparing the errorbars in the far red and blue wings of each lines, we find that the errorbars are similar. Larger errorbars can also be seen in the central part of the $\mathrm{H} \alpha$ due to the narrow line subtraction. Figure 9 shows the distributions of the errorbars as a function of the line flux for segments ha \pm 5 , ha0, and ha \pm 1 . It can be seen that in the case of ha0 and ha+1, there is a slight anticorrelation with flux and two points, corresponding to very low flux $\left(F<4 \times 10^{-13} \mathrm{erg} \mathrm{cm}^{-2} \mathrm{~s}^{-1}\right.$ in 2005), have the highest errorbar of $(40-70) \%$.

We constructed light curves for each segment of the $\mathrm{H} \alpha$ and $\mathrm{H} \beta$ lines. Figure 10 presents light curves of profile segments in approximately identical velocity intervals in the blue and red line wings (segments from 1 to 5, where a higher number corresponds to a higher velocity, see Table 3 ) and for the central part ( 0 in Table 3 or $\mathrm{H} \alpha \_c, H \beta \_c$ in Fig. 10 , corresponding to the interval $\sim \pm 500 \mathrm{~km} \mathrm{~s}^{-1}$ ). To compare the segment variation with the continuum, we plot (as a solid line) the continuum flux variation in the central part (see Fig. 10).

In 1996-1997, the blue segments 2 and 3 were slightly brighter than the red ones, while the segments 1,4 , and 5 were similar to the red ones. In 1998-2001, the blue segments 4 and 5 (3500-5500 $\mathrm{km} \mathrm{s}^{-1}$, which are the regions the closest to the $\mathrm{BH}$ ) and the segment 3 (in 2002-2006) of both lines were essentially fainter than the red ones. In 1998-2004, the blue segments 1 and $2\left(500-3500 \mathrm{~km} \mathrm{~s}^{-1}\right)$ were close to the corresponding red ones or slightly fainter (see Fig. 10).

\subsection{The line-segment to line-segment flux and continuum-line-segment relations}

The line-segment to line-segment flux and continuum-linesegment relations for the $\mathrm{H} \alpha$ and $\mathrm{H} \beta$ lines are practically identical, therefore here we present only results for the $\mathrm{H} \alpha$ line. First, we search for relations between the $\mathrm{H} \alpha$ segments that are symmetric with respect to the center (i.e., segments -1 , and $1,-2$ and $2, \ldots-5$ and 5). In Fig. 11, we present the response of symmetrical segments of $\mathrm{H} \alpha$ to each other for the three periods given in Paper I. As can be seen from Fig. 11, the symmetric segments are quite strongly correlated, with some notable exceptions: a) weaker response of the red to the blue wing in the II and III period and b) the apparent bifurcation in the Ha2/Ha-2 and Ha3/Ha-3 plots. This appears to be associated with Period III, related to the appearance of the $3000 \mathrm{~km} \mathrm{~s}^{-1}$ "red bump". This also implies that lines are probably formed in a multi-component BLR and that the geometry of the BLR changed during the monitoring period. 

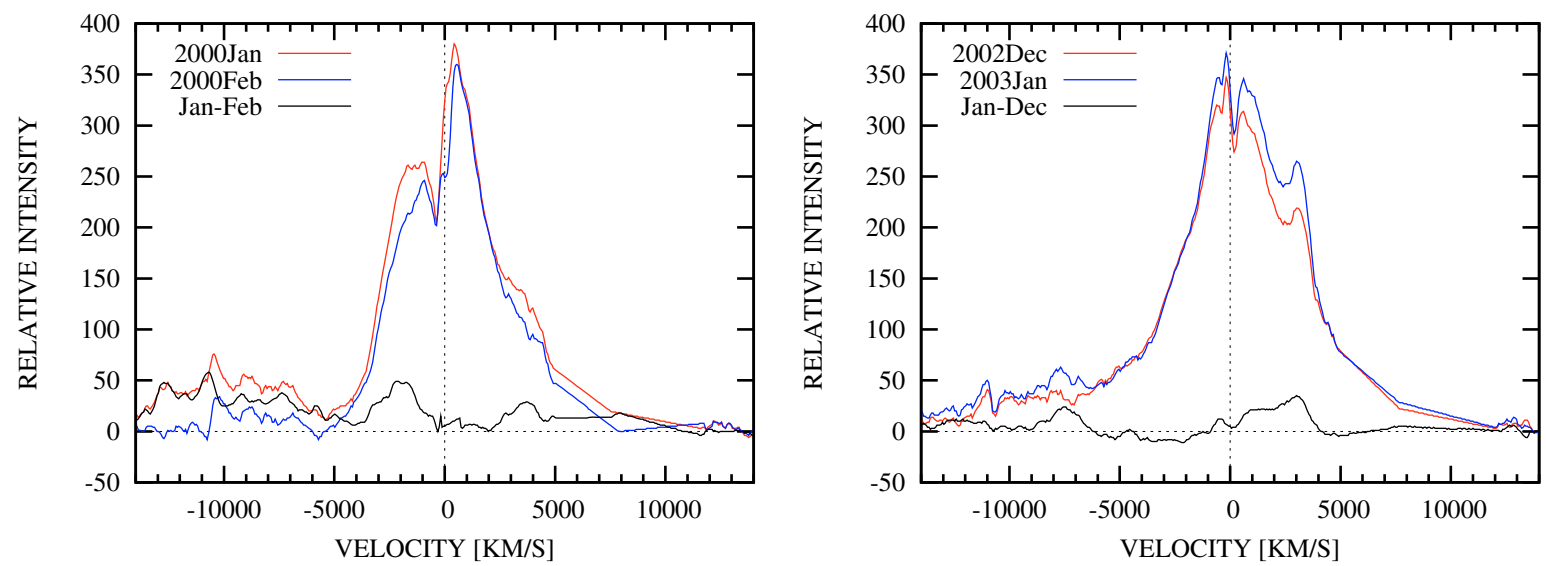

Fig. 5. The $\mathrm{H} \beta$ broad emission lines of NGC 4151 in particular for two consecutive months where bumps were strong (red and blue line). Their residual is also given (black line below).

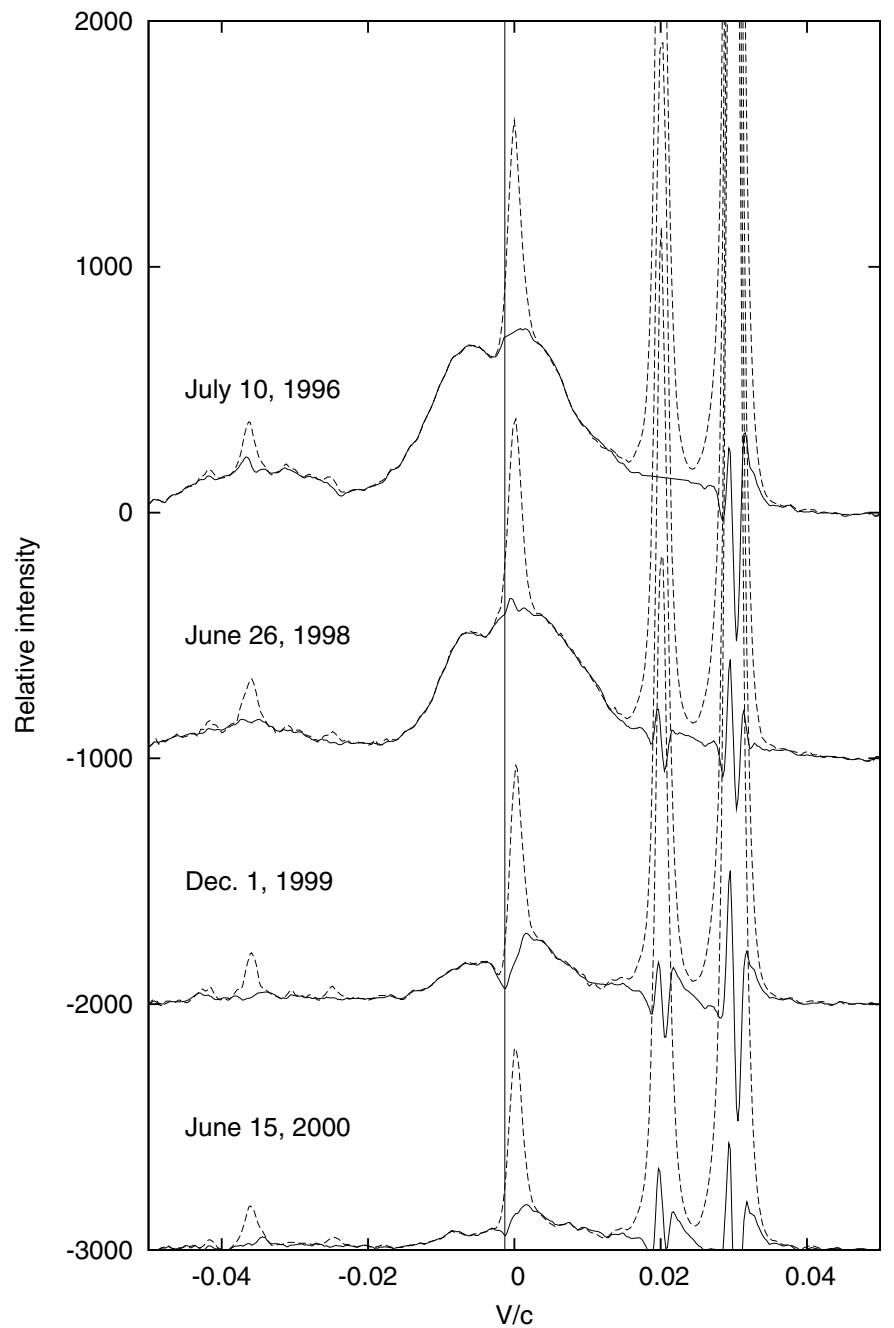

Fig. 6. The absorption seen in years 1996, 1998, 1999, and 2000 (from top to bottom). The $\mathrm{H} \beta$ line after subtraction of the narrow lines is shown with solid lines. The vertical line indicates the radial velocity of $-400 \mathrm{~km} \mathrm{~s}^{-1}$.

Additionally, in Fig. 12 we present the response of different $\mathrm{H} \alpha$ segments to the continuum flux. As can be seen, the response differ: in the far wings, the response to the continuum is almost linear for the red wing (segments 4 and 5) and for a fraction of the blue wing (segment -4), but for the far blue wing ( -5500 to $-4500 \mathrm{~km} \mathrm{~s}^{-1}$ ), there is practically no response for $F_{\mathrm{c}}<7 \times 10^{-14} \mathrm{erg} \mathrm{cm}^{-2} \mathrm{~s}^{-1} \AA^{-1}$. For higher continuum fluxes, the far blue wing has a higher flux, but it also seems that there is no linear relation between the line wing and the continuum flux. On the other hand, the central segments (from -3500 to $3500 \mathrm{~km} \mathrm{~s}^{-1}$ ) have a response to the continuum similar to that of the $\mathrm{H} \beta$ and $\mathrm{H} \alpha$ total line fluxes (see Paper I) - a linear response for the low continuum flux $\left(F_{\mathrm{c}}<7 \times 10^{-14} \mathrm{erg} \mathrm{cm}^{-2} \mathrm{~s}^{-1} \AA^{-1}\right)$ and no linear response for the high continuum flux $\left(F_{\mathrm{c}}>7 \times 10^{-14} \mathrm{erg} \mathrm{cm}^{-2} \mathrm{~s}^{-1} \AA^{-1}\right)$. The linear response indicates that this part of the line (red wings and central segments at the low continuum flux) originates in the part of the BLR ionized by the AGN source, while the blue and partly central part of the line could partly originate in a substructure outside this BLR (and probably not photoionized). We note here that the photoionization in the case of a mix of a thin (fully ionized) and thick (ionization-bounded) clouds can explain the observed non-linear response of emission lines to the continuum in variable as seen in the central parts of broad lines (see Shields et al. 1995), i.e., in the case of the optically thick BLR the detailed photoionization models show that the response of the Balmer lines declines as the continuum flux increases (see Goad et al. 2004; Korista \& Goad 2004). In our case, we found that the flux in the wings (except the far blue wing) has an almost linear response to the continuum flux, while the central parts have a non-linear response to the higher continuum flux. The response to the continuum flux of the far blue wing $(-5)$ and far red wing $(+5)$ is also very different, which may be indicative of different physical conditions in subregions or across the BLR.

In Fig. 13, the flux of different $\mathrm{H} \alpha$ segments as a function of the flux of the central segment $(\mathrm{H} \alpha 0)$ are presented. It can be seen that there are different relations between different $\mathrm{H} \alpha$ segments and $\mathrm{H} \alpha 0$ : for segments close to the center ( $\mathrm{H} \alpha-1, \mathrm{H} \alpha-2$, $\mathrm{H} \alpha 1$, and $\mathrm{H} \alpha 2$ ) the relation is almost linear, indicating that the core of the line originates in the same substructures (Fig. 13); segments in the $\mathrm{H} \alpha$ wings (the near blue wing $\mathrm{H} \alpha-3$ and the red wing $\mathrm{H} \alpha 3, \mathrm{H} \alpha 4$, and $\mathrm{H} \alpha 5$ ) also show a linear response to the central segment (but the scatter of the points is larger than in the previous case), which also indicates that a portion of the emission in the center and in these segments originates in the same emission region. In contrast, the far blue wing ( $\mathrm{H} \alpha-4$ and $\mathrm{H} \alpha-$ 5 ) responds weakly to the variation in the line center, especially $\mathrm{H} \alpha-5$, which exhibits practically independent variations with respect to the central segment. 


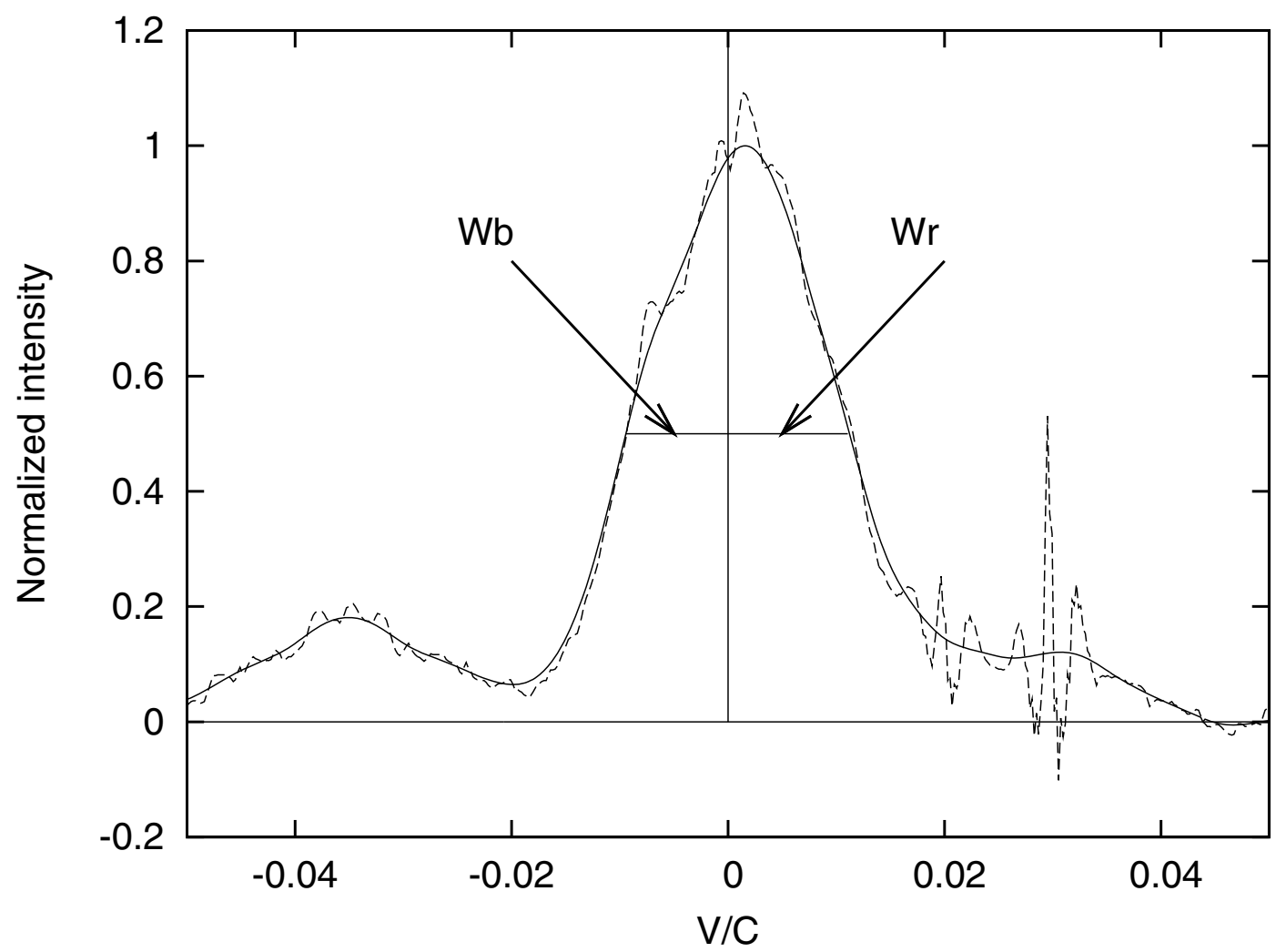

Fig. 7. An example of the FWHM and asymmetry measurements. The observed spectra is denoted by the dashed line and the smoothed spectra by the solid line.

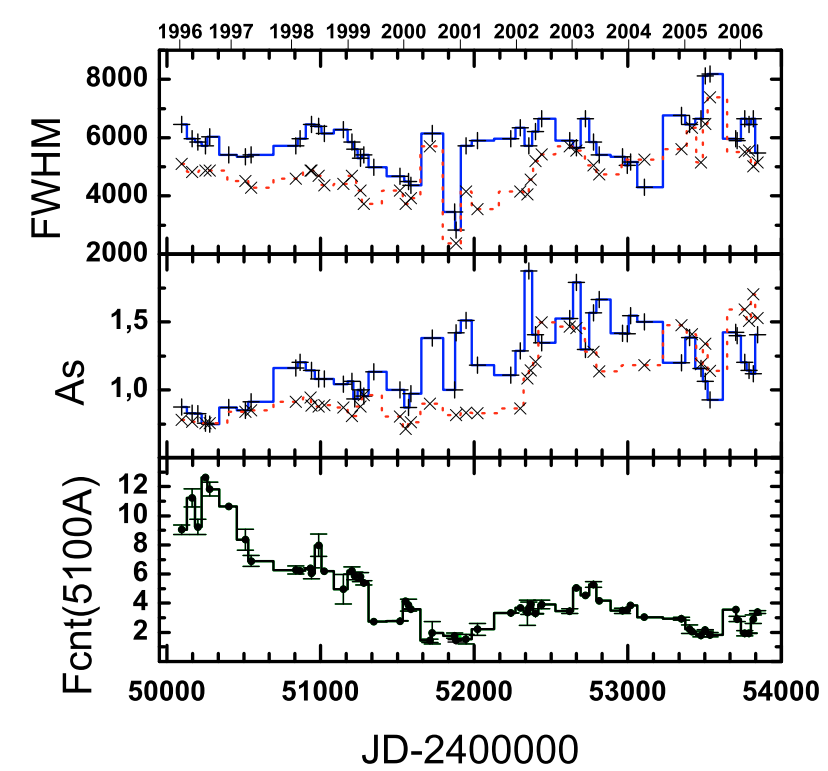

Fig. 8. Variations in the FWHM (upper panel), asymmetry (middle panel) in $\mathrm{H} \alpha$ (denoted with crosses) and $\mathrm{H} \beta$ (denoted with plus) broad lines, and of the continuum flux at $\lambda 5100 \AA$ (bottom panel) in 1996-2006. The abscissae shows the Julian date (bottom) and the corresponding year (up). The continuum flux is given in $10^{-14} \mathrm{erg} \mathrm{cm}^{-2} \mathrm{~s}^{-1} \AA^{-1}$.

\section{The Balmer decrement}

\subsection{Integral Balmer decrement}

From monthly-averaged profiles of the broad $\mathrm{H} \alpha$ and $\mathrm{H} \beta$ lines, we determined their integrated fluxes to be in the range between -5500 and $+5500 \mathrm{~km} \mathrm{~s}^{-1}$ in radial velocity. We call the "integral Balmer decrement" (BD), the integrated flux ratio $F(\mathrm{H} \alpha) / F(\mathrm{H} \beta)$.

Figure 14 shows the behavior of the integrated BD (upper panel) and continuum flux at the wavelength $5100 \AA$ (bottom panel). In 1999-2006, an anticorrelation between the changes of the integrated BD and continuum flux was observed, which was especially noticeable in 1999-2001.

Table 5 presents yearly-averaged values of the BD and continuum flux determined from monthly-averaged profiles for each year.

We found that in 1996-1998 the continuum flux was high and varied within the limits given by $F_{\mathrm{c}} \sim(6-12) \times$ $10^{-14} \mathrm{erg} \mathrm{cm}^{-2} \mathrm{~s}^{-1} \AA^{-1}$, but the BD was practically unchanged. In Paper I, we already noted the absence of correlation between the continuum flux and the integrated flux of the broad lines for the above-mentioned flux values. We also found (see Fig. 14) that the Balmer decrement is systematically higher in 1999-2001.

Table 5 also provides the average data of the BD and continuum flux for the periods of 1996-1998, 1999, 2000-2001, and 2002-2006. It is evident that in the period of 1996-1998 the BD did not vary within the error bars in spite of strong variations in the continuum. Radical changes (the increase in the BD) started in 1999.

The BD reached its maximum in 2000-2001. In 2002-2006, the average values of the $\mathrm{BD}$ then coincided with those of 1996-1998. Thus, it can be concluded with confidence that from 1999 to 2001 we observed an obvious increase in the BD. 
Table 2. The FWHM and asymmetry of the $\mathrm{H} \alpha$ and $\mathrm{H} \beta$ broad emission lines in the period of 1996-2006.

\begin{tabular}{|c|c|c|c|c|c|c|}
\hline "Year_month & $\begin{array}{c}\text { JD } \\
2400000+\end{array}$ & $\begin{array}{c}F W H M \mathrm{H} \beta \pm \sigma \\
\mathrm{km} \mathrm{s}^{-1}\end{array}$ & $\overline{A \mathrm{AH} \beta \pm \sigma}$ & $\begin{array}{c}F W H M \mathrm{H} \alpha \pm \sigma \\
\mathrm{km} \mathrm{s}^{-1}\end{array}$ & $\overline{A \mathrm{AH} \alpha \pm \sigma}$ & $\begin{array}{c}F(5100) \pm \sigma \\
10^{-14} \mathrm{erg} \mathrm{cm}^{-2} \mathrm{~s}^{-1} \AA^{-1}\end{array}$ \\
\hline 1 & 2 & 3 & 4 & 5 & 6 & 7 \\
\hline 1996b_01 & 50096.0 & $6860 \pm 566$ & $0.904 \pm 0.043$ & $5332 \pm 324$ & $0.799 \pm 0.030$ & $9.031 \pm 0.322$ \\
\hline 1996b_03 & 50163.4 & $6182 \pm 303$ & $0.877 \pm 0.069$ & $5036 \pm 291$ & $0.782 \pm 0.021$ & $11.219 \pm 0.617$ \\
\hline 1996b_04 & 50200.8 & $6183 \pm 479$ & $0.877 \pm 0.072$ & - & -- & $9.226 \pm 0.527$ \\
\hline 1996b_06 & 50249.3 & $6028 \pm 434$ & $0.797 \pm 0.060$ & - & - & $12.641-$ \\
\hline 1996b_07 & 50277.8 & $6367 \pm 479$ & $0.783 \pm 0.048$ & $5058 \pm 259$ & $0.762 \pm 0.011$ & $11.822 \pm 0.473$ \\
\hline 1996b_11 & 50402.6 & $5659 \pm 348$ & $0.896 \pm 0.035$ & - & - & $10.649-$ \\
\hline 1997b_03 & 50510.9 & $5568 \pm 305$ & $0.884 \pm 0.048$ & $4808 \pm 420$ & $0.853 \pm 0.019$ & $8.350 \pm 0.716$ \\
\hline 1997b_04 & 50547.9 & $5567 \pm 218$ & $0.946 \pm 0.046$ & $4512 \pm 323$ & $0.854 \pm 0.005$ & $6.897 \pm 0.375$ \\
\hline 1996-1997 & & $6051 \pm 449$ & $0.871 \pm 0.054$ & $4959 \pm 307$ & $0.810 \pm 0.042$ & $9.979 \pm 1.950$ \\
\hline 1998b_01 & 50838.0 & $5844 \pm 173$ & $1.159 \pm 0.005$ & $4831 \pm 323$ & $0.947 \pm 0.050$ & $6.280 \pm 0.277$ \\
\hline 1998b_02 & 50867.4 & $6059 \pm 130$ & $1.189 \pm 0.021$ & - & - & $6.219-$ \\
\hline 1998r_04 & 50934.5 & - & - & $5058 \pm 259$ & $0.981 \pm 0.051$ & $6.387-$ \\
\hline 1998b_05 & 50940.4 & $6489 \pm 42$ & $1.131 \pm 0.016$ & $5150 \pm 388$ & $0.933 \pm 0.071$ & $6.049 \pm 0.385$ \\
\hline 1998b_06 & 50988.3 & $6458 \pm 86$ & $1.059 \pm 0.029$ & $4922 \pm 323$ & $0.931 \pm 0.073$ & $7.974 \pm 0.776$ \\
\hline 1998b_07 & 51025.3 & $6214 \pm 88$ & $1.082 \pm 0.000$ & $4762 \pm 549$ & $0.935 \pm 0.067$ & $6.205-$ \\
\hline 1998_11-12 & 51148.6 & $6275 \pm 1$ & $1.062 \pm 0.028$ & $4694 \pm 387$ & $0.909 \pm 0.053$ & $4.975 \pm 1.011$ \\
\hline 1998 & & $6223 \pm 245$ & $1.114 \pm 0.054$ & $4903 \pm 176$ & $0.939 \pm 0.024$ & $6.298 \pm 0.880$ \\
\hline 1999b_01 & 51202.6 & $5967 \pm 174$ & $1.063 \pm 0.001$ & $4831 \pm 194$ & $0.863 \pm 0.079$ & $6.245 \pm 0.243$ \\
\hline 1999b_02 & 51223.1 & $5721 \pm 174$ & $0.957 \pm 0.031$ & - & - & $5.873 \pm 0.258$ \\
\hline 1999b_03 & 51260.5 & $5475 \pm 261$ & $1.045 \pm 0.064$ & $4398 \pm 291$ & $0.910 \pm 0.045$ & $5.793 \pm 0.296$ \\
\hline 1999b_04 & 51281.5 & $5567 \pm 218$ & $1.011 \pm 0.078$ & $4010 \pm 388$ & $0.959 \pm 0.001$ & $5.397 \pm 0.146$ \\
\hline 1999b_06 & 51346.4 & $5352 \pm 522$ & $1.173 \pm 0.058$ & - & - & $2.753-$ \\
\hline 1999b_12 & 51515.6 & $5106 \pm 609$ & $1.023 \pm 0.032$ & $4352 \pm 226$ & $0.836 \pm 0.045$ & $2.782 \pm 0.016$ \\
\hline 1999 & & $5531 \pm 298$ & $1.045 \pm 0.072$ & $4903 \pm 337$ & $0.892 \pm 0.054$ & $4.807 \pm 1.603$ \\
\hline 2000b_01 & 51553.6 & - & - & $4010 \pm 388$ & $0.744 \pm 0.041$ & $4.155-$ \\
\hline 2000_1_2 & 51571.6 & $4890 \pm 566$ & $0.960 \pm 0.124$ & - & - & $3.868 \pm 0.406$ \\
\hline 2000b_ō & 51589.5 & $4767 \pm 566$ & $1.036 \pm 0.090$ & $4056 \pm 194$ & $0.801 \pm 0.055$ & $3.581 \pm 0.029$ \\
\hline 2000b_06 & 51711.3 & - & - & $6244 \pm 775$ & $1.014 \pm 0.162$ & $1.434 \pm 0.115$ \\
\hline 2000_6_7 & 51728.6 & $6336 \pm 262$ & $1.423 \pm 0.060$ & - & - & $1.974 \pm 0.764$ \\
\hline 2000b_11 & 51874.1 & $3968 \pm 739$ & $1.014 \pm 0.020$ & - & - & $1.778 \pm 0.177$ \\
\hline 2000b_12 & 51883.6 & $3383 \pm 783$ & $1.353 \pm 0.096$ & $2985 \pm 871$ & $0.805 \pm 0.013$ & $1.427 \pm 0.09$ \\
\hline 2001b_02 & 51947.9 & $6367 \pm 914$ & $1.523 \pm 0.015$ & $4603 \pm 646$ & $0.907 \pm 0.106$ & $1.538 \pm 0.320$ \\
\hline 2001b_11 & 52238.0 & $6152 \pm 261$ & $1.127 \pm 0.027$ & - & - & $3.331 \pm 0.007$ \\
\hline $2000-2001$ & & $5123 \pm 1199$ & $1.205 \pm 0.224$ & $4380 \pm 1195$ & $0.854 \pm 0.107$ & $2.565 \pm 1.193$ \\
\hline 2002b_01 & 52299.4 & $6183 \pm 217$ & $1.284 \pm 0.006$ & $4785 \pm 903$ & $0.957 \pm 0.134$ & $3.680-$ \\
\hline 2002r_03 & 52345.8 & - & - & $4603 \pm 775$ & $1.173 \pm 0.124$ & $3.348 \pm 0.862$ \\
\hline 2002_3_4 & 52357.3 & $6060 \pm 479$ & $1.706 \pm 0.239$ & - & - & $3.645 \pm 0.420$ \\
\hline $2002 r \_04$ & 52368.7 & - & - & $5150 \pm 838$ & $1.196 \pm 0.076$ & $3.942-$ \\
\hline 2002b_05 & 52398.7 & $6336 \pm 173$ & $1.369 \pm 0.050$ & $5605 \pm 581$ & $1.203 \pm 0.005$ & $3.283 \pm 0.021$ \\
\hline 2002b_06 & 52439.0 & $7012 \pm 521$ & $1.285 \pm 0.088$ & $5582 \pm 226$ & $1.414 \pm 0.115$ & $3.903 \pm 0.292$ \\
\hline 2002b_12 & 52621.0 & $6121 \pm 305$ & $1.489 \pm 0.051$ & $5970 \pm 387$ & $1.374 \pm 0.129$ & $3.467 \pm 0.157$ \\
\hline 2002 & & $6490 \pm 465$ & $1.427 \pm 0.177$ & $5283 \pm 528$ & $1.220 \pm 0.163$ & $3.629 \pm 0.243$ \\
\hline 2003b_01 & 52665.9 & $5905 \pm 348$ & $1.710 \pm 0.112$ & $5651 \pm 129$ & $1.373 \pm 0.117$ & $5.072-$ \\
\hline 2003b_03 & 52723.8 & $6336 \pm 434$ & $1.344 \pm 0.066$ & - & - & $4.541-$ \\
\hline 2003b_05 & 52777.1 & $5906 \pm 87$ & $1.559 \pm 0.009$ & $5287 \pm 324$ & $1.260 \pm 0.027$ & $5.283 \pm 0.205$ \\
\hline 2003b_06 & 52813.7 & $5660 \pm 349$ & $1.630 \pm 0.050$ & $5013 \pm 388$ & $1.163 \pm 0.038$ & $4.184-$ \\
\hline 2003b_11 & 52966.8 & $5505 \pm 217$ & $1.358 \pm 0.082$ & - & - & $3.478 \pm 0.193$ \\
\hline 2003b_12 & 52995.6 & $5414 \pm 523$ & $1.352 \pm 0.083$ & - & - & $3.515-$ \\
\hline 2003 & & $5788 \pm 336$ & $1.492 \pm 0.162$ & $5317 \pm 320$ & $1.265 \pm 0.105$ & $4.346 \pm 0.763$ \\
\hline 2004b_01 & 53019.5 & $5351 \pm 261$ & $1.456 \pm 0.124$ & - & - & $3.851-$ \\
\hline 2004b_12 & 53349.0 & $6889 \pm 173$ & $1.240 \pm 0.057$ & $5879 \pm 389$ & $1.422 \pm 0.076$ & $2.932 \pm 0.105$ \\
\hline 2004 & & $6120 \pm 1088$ & $1.348 \pm 0.153$ & $5879 \pm 389$ & $1.422 \pm 0.076$ & $3.392 \pm 0.650$ \\
\hline 2005_1_2 & 53402.8 & $7013 \pm 783$ & $1.332 \pm 0.076$ & - & - & $2.218 \pm 0.308$ \\
\hline 2005b_02 & 53417.5 & - & - & $6586 \pm 356$ & $1.359 \pm 0.072$ & $2.040-$ \\
\hline 2005b_05 & 53505.3 & $8305 \pm 262$ & $1.093 \pm 0.043$ & $6882 \pm 582$ & $1.265 \pm 0.105$ & $2.140 \pm 0.037$ \\
\hline 2005b_06 & 53535.0 & $8427 \pm 348$ & $0.957 \pm 0.041$ & $7635 \pm 357$ & $1.100 \pm 0.058$ & $1.841 \pm 0.142$ \\
\hline 2005b_11 & 53704.0 & $6059 \pm 130$ & $1.402 \pm 0.031$ & - & - & $3.567-$ \\
\hline 2005b_12 & 53711.5 & $5967 \pm 88$ & $1.395 \pm 0.007$ & - & - & $2.911 \pm 0.177$ \\
\hline
\end{tabular}


Table 2. continued.

\begin{tabular}{ccccccc}
\hline \hline Year_month & $\begin{array}{c}\mathrm{JD} \\
2400000+\end{array}$ & $\begin{array}{c}F W H M \mathrm{H} \beta \pm \sigma \\
\mathrm{km} \mathrm{s}^{-1}\end{array}$ & $A \mathrm{H} \beta \pm \sigma$ & $\begin{array}{c}F W H M \mathrm{H} \alpha \pm \sigma \\
\mathrm{km} \mathrm{s}^{-1}\end{array}$ & $A \mathrm{H} \alpha \pm \sigma$ & $\begin{array}{c}F(5100) \pm \sigma \\
10^{-14} \mathrm{erg} \mathrm{cm}^{-2} \mathrm{~s}^{-1} \AA^{-1}\end{array}$ \\
\hline 1 & 2 & 3 & 4 & 5 & 6 & 7 \\
\hline 2005 & & $7154 \pm 1180$ & $1.236 \pm 0.200$ & $7034 \pm 540$ & $1.241 \pm 0.131$ & $2.953 \pm 0.656$ \\
\hline 2006b_01 & 53762.4 & $6797 \pm 218$ & $1.188 \pm 0.021$ & $6015 \pm 710$ & $1.449 \pm 0.202$ & $1.939 \pm 0.179$ \\
2006b_02 & 53788.0 & $6582 \pm 174$ & $1.161 \pm 0.027$ & $5925 \pm 518$ & $1.403 \pm 0.145$ & $1.926 \pm 0.016$ \\
2006b_03 & 53816.9 & $6704 \pm 86$ & $1.117 \pm 0.002$ & $5446 \pm 613$ & $1.546 \pm 0.222$ & $2.894 \pm 0.293$ \\
2006b_04 & 53845.1 & $5691 \pm 305$ & $1.373 \pm 0.044$ & $5401 \pm 356$ & $1.480 \pm 0.069$ & $3.390 \pm 0.103$ \\
\hline 2006 & & $6444 \pm 509$ & $1.210 \pm 0.113$ & $5697 \pm 318$ & $1.470 \pm 0.060$ & $2.537 \pm 0.727$ \\
\hline mean 96-06 & $6021 \pm 851$ & $1.187 \pm 0.237$ & $5164 \pm 873$ & $1.072 \pm 0.245$ & \\
\hline Paper I & $6110 \pm 440$ & $1.056 \pm 0.018$ & $4650 \pm 420$ & $1.00 \pm 0.023$ & \\
\hline
\end{tabular}

Columns: 1 - year and month (e.g., 1996_01 = 1996_January); 2 - Julian date (JD); 3 - the $F W H M$ of H $\beta ; 4-$ the asymmetry of H $\beta$; 5 - the $F W H M$ of $\mathrm{H} \alpha ; 6$ - the asymmetry of $\mathrm{H} \alpha ; 7-F(5100)$, the continuum flux at $\lambda 5100 \AA$ in units of $10^{-14} \mathrm{erg} \mathrm{cm}^{-2} \mathrm{~s}^{-1} \AA^{-1}$ and $\sigma(\mathrm{cnt})$, the estimated continuum flux error in the same units.

Table 3. The beginning and end radial velocities, $V_{\text {beg }}$ and $V_{\text {end }}$, in $\mathrm{km} \mathrm{s}^{-1}$ for different segments of the line profiles.

\begin{tabular}{|c|c|c|c|c|c|c|c|c|c|c|c|}
\hline$\overbrace{V_{r}}$ Segment & -5 & -4 & -3 & -2 & -1 & $0(\mathrm{C})$ & +1 & +2 & +3 & +4 & +5 \\
\hline$V_{\text {beg }}$ & -5500 & -4500 & -3500 & -2500 & -1500 & -500 & 500 & 1500 & 2500 & 3500 & 4500 \\
\hline$V_{\text {end }}$ & -4500 & -3500 & -2500 & -1500 & -500 & 500 & 1500 & 2500 & 3500 & 4500 & 5500 \\
\hline
\end{tabular}

\subsection{Balmer decrement of different profile segments}

From monthly-averaged profiles of $\mathrm{H} \alpha$ and $\mathrm{H} \beta$, we determined the BD for the segments of Table 3. Figure 15 shows the BD variation in a $2 \mathrm{D}$ plane (year- $V_{r}$ ), and Fig. 16 indicates the changes in the $\mathrm{BD}$ of different segments of the profiles during the whole monitoring period.

It can be seen from Figs. 15 and 16 that in 2000-2001 (JD = 51 200-52 300) the values of the BD in different segments were always on average noticeably larger than in the other years, and the blue wing had always on average a noticeably larger BD than the red one. In 1996-1998, BDs of the blue and red wings practically coincided, and in 2002-2006, they either coincided or the BD of the blue wing was slightly larger. During the entire monitoring period, the value of the $\mathrm{BD}$ along the line profile in central segments (in segments $0, \pm 1$ corresponding to the velocity range from $-1500 \mathrm{~km} \mathrm{~s}^{-1}$ to $1500 \mathrm{~km} \mathrm{~s}^{-1}$ ) were considerably larger (by $\sim 1.5$ ) on average than at the periphery (segments from \pm 2 to \pm 5 corresponding to the velocity range from $\left((-5500)-(-1500) \mathrm{km} \mathrm{s}^{-1}\right)$ to $\left.\left.(+5500)-(+1500) \mathrm{km} \mathrm{s}^{-1}\right)\right)$.

\subsubsection{Balmer decrement variations as a function of the radial velocity}

As an illustration of the variation in the Balmer decrement with the radial velocity during the monitoring period, we show in Fig. 17 the BD as a function of the radial velocity. Since the $\mathrm{BD}$ versus velocity remains the same throughout a year, we provide a few examples only for monthly-averaged spectra for each year of the monitoring period.

On the other hand, the behavior of the Balmer decrement as a function of the radial velocity differs between different years. As a rule, in 1996-1998 the maximum of the BD occurred at $\sim 1000 \mathrm{~km} \mathrm{~s}^{-1}$, while the BD slowly decreased in the velocity range from 0 to $1000 \mathrm{~km} \mathrm{~s}^{-1}$, and sharply decreased in the region $> \pm 1000 \mathrm{~km} \mathrm{~s}^{-1}$, usually more strongly in the region of negative velocity. We recall here the results obtained from the photoionization model of Korista \& Goad (2004) where they found velocity-dependent variations in the Balmer decrement.
They found that the Balmer decrement is steeper in the line core than in line wings, as we obtained in some cases (see Fig. 17), but it is interesting that in all periods this peak is offset from the central part to the blue side and also that there are several cases where the Balmer decrement is steeper in the wings. On the other hand, if the velocity field is dominated by a central massive object, one can expect to observe a symmetrical BD in the blue and red part of velocity field (Korista \& Goad 2004). In our case, we obtained different asymmetrical shapes of the BD versus velocity field. The BD seems to have a systematic change in behavior starting around 2002 - i.e., corresponding to period III, when the "red bump" appears, showing two maxima in the BD versus velocity field. This may indicate that the velocity field of the BLR is not dominated by a central massive black hole, i.e., that it favors some kind of stream of the emitting material such as e.g., outflow or inflow.

In 1999-2001, the maximum values of the $\mathrm{BD} \sim(6-8)$ were observed in the velocity region $\pm 1000 \mathrm{~km} \mathrm{~s}^{-1}$, while a steeper decrease in the $\mathrm{BD}$ was more often observed in the region of the positive velocity. The change in the BD relative to the radial velocity in 2002-2006 differed strongly from those in 1996-2001. In these years, 2 peaks (bumps) were observed in the BD distribution: one at radial velocities from -2000 to -1000 with larger values of the $\mathrm{BD}$, and a second at $+3000 \mathrm{~km} \mathrm{~s}^{-1}$ with somewhat smaller (by $\sim(0.5-1.0)$ ) values of the BD.

\subsection{Balmer decrement and helium line ratio}

To probe the physical conditions of the BLR, we studied the flux ratio of He II $\lambda 4686$ and HeI $\lambda 5876$ broad lines. From all the available spectra, we selected only 21 spectra where the broad helium lines could be precisely measured, and where the two helium lines were observed during the same night. In the case of minimum activity, we note the broad component of the He II $\lambda 4686$ line could not be detected at all.

We use the helium lines He II $\lambda 4686$ and He I $\lambda$ 5876, since these two lines originates from two different ionization stages and, thus, are very sensitive to changes in the electron temperature and density of the emitting region (Griem 1997). 
Table 4. The errors in measurements (e $\pm \sigma$ ) for all line segments (see Table 3) of $\mathrm{H} \alpha$ and $\mathrm{H} \beta$ given in percent. For each segment, the mean-year flux is also given in units $10^{-13} \mathrm{erg} \mathrm{cm}^{-2} \mathrm{~s}^{-1}$.

\begin{tabular}{ccccccccccccc}
\hline \hline Year & \multicolumn{3}{c}{$\mathrm{Ha}(-5)$} & \multicolumn{2}{c}{$\mathrm{Ha}(+5)$} & \multicolumn{3}{c}{$\mathrm{Ha}(-4)$} & \multicolumn{3}{c}{$\mathrm{Ha}(+4)$} & \multicolumn{3}{c}{$\mathrm{Ha}(-3)$} & \multicolumn{3}{c}{$\mathrm{Ha}(+3)$} \\
& Flux & $(e \pm \sigma)$ & Flux & $(e \pm \sigma)$ & Flux & $(e \pm \sigma)$ & Flux & $(e \pm \sigma)$ & Flux & $(e \pm \sigma)$ & Flux & $(e \pm \sigma)$ \\
\hline 1996 & 6.327 & $9.6 \pm 9.7$ & 7.052 & $13.3 \pm 10.5$ & 12.456 & $9.9 \pm 5.9$ & 12.494 & $11.7 \pm 7.1$ & 22.736 & $9.1 \pm 6.8$ & 17.722 & $10.9 \pm 7.1$ \\
1997 & 3.904 & $11.1 \pm 6.1$ & 4.950 & $9.7 \pm 0.8$ & 8.100 & $6.7 \pm 8.8$ & 10.048 & $9.7 \pm 3.8$ & 18.741 & $6.7 \pm 9.2$ & 16.634 & $9.1 \pm 5.3$ \\
1998 & 2.497 & $11.7 \pm 4.2$ & 4.516 & $7.2 \pm 6.6$ & 6.381 & $5.4 \pm 2.4$ & 8.904 & $7.4 \pm 4.8$ & 16.570 & $3.2 \pm 2.9$ & 16.518 & $4.6 \pm 4.7$ \\
1999 & 2.030 & $14.3 \pm 18.3$ & 5.133 & $6.2 \pm 3.8$ & 5.649 & $2.1 \pm 3.0$ & 8.776 & $3.3 \pm 1.1$ & 16.851 & $2.2 \pm 2.0$ & 16.470 & $2.1 \pm 1.0$ \\
2000 & 1.647 & $5.3 \pm 4.3$ & 3.460 & $4.3 \pm 3.5$ & 3.561 & $4.4 \pm 4.4$ & 5.570 & $4.2 \pm 3.5$ & 9.204 & $4.2 \pm 3.8$ & 7.770 & $3.8 \pm 3.6$ \\
2001 & 2.251 & $5.5 \pm 5.3$ & 2.906 & $7.3 \pm 6.2$ & 3.671 & $4.7 \pm 6.0$ & 5.220 & $5.9 \pm 6.1$ & 7.249 & $7.3 \pm 6.0$ & 7.760 & $8.1 \pm 7.0$ \\
2002 & 3.686 & $3.5 \pm 0.4$ & 3.016 & $3.0 \pm 1.3$ & 5.041 & $2.7 \pm 2.4$ & 6.443 & $4.9 \pm 5.3$ & 7.792 & $3.6 \pm 1.4$ & 10.742 & $4.1 \pm 5.1$ \\
2003 & 3.004 & $5.7 \pm 5.0$ & 4.186 & $2.9 \pm 1.7$ & 4.447 & $4.9 \pm 2.4$ & 7.560 & $3.4 \pm 1.3$ & 9.104 & $4.0 \pm 2.3$ & 14.034 & $2.9 \pm 2.1$ \\
2004 & 3.261 & $3.2 \pm 0.8$ & 2.722 & $3.3 \pm 1.8$ & 4.340 & $6.1 \pm 2.3$ & 5.299 & $1.9 \pm 1.3$ & 6.619 & $5.7 \pm 2.4$ & 9.922 & $3.9 \pm 2.1$ \\
2005 & 2.908 & $4.3 \pm 1.3$ & 2.350 & $7.1 \pm 6.7$ & 4.122 & $4.4 \pm 3.4$ & 4.687 & $5.9 \pm 6.2$ & 5.756 & $4.5 \pm 3.1$ & 8.575 & $5.5 \pm 4.3$ \\
2006 & 2.906 & $1.7 \pm 0.4$ & 1.938 & $1.7 \pm 1.7$ & 3.979 & $3.0 \pm 2.7$ & 4.115 & $3.0 \pm 1.9$ & 5.784 & $2.7 \pm 2.8$ & 10.459 & $1.9 \pm 1.3$ \\
\hline mean & $6.9 \pm 4.1$ & \multicolumn{4}{c}{$6.0 \pm 3.4$} & & $4.9 \pm 2.2$ & & $5.6 \pm 3.0$ & & $4.8 \pm 2.1$ & $5.2 \pm 3.0$ \\
\hline
\end{tabular}

\begin{tabular}{|c|c|c|c|c|c|c|c|c|c|c|c|c|}
\hline \multirow[t]{2}{*}{ Year } & \multicolumn{2}{|c|}{$\mathrm{Hb}(-5)$} & \multicolumn{2}{|c|}{$\mathrm{Hb}(+5)$} & \multicolumn{2}{|c|}{$\mathrm{Hb}(-4)$} & \multicolumn{2}{|c|}{$\mathrm{Hb}(+4)$} & \multicolumn{2}{|c|}{$\mathrm{Hb}(-3)$} & \multicolumn{2}{|c|}{$\mathrm{Hb}(+3)$} \\
\hline & Flux & $(e \pm \sigma)$ & Flux & $(e \pm \sigma)$ & $\operatorname{lux}$ & $(e \pm \sigma)$ & Flux & $(e \pm \sigma)$ & Flux & $(e \pm \sigma)$ & Flux & $(e \pm \sigma)$ \\
\hline 1996 & .511 & $3.2 \pm 0.17$ & $\overline{535}$ & $6.5 \pm 5.3$ & 4.965 & $7.0 \pm 5$ & $\overline{70}$ & $7.1 \pm 1.5$ & .003 & $7.6 \pm 6.1$ & .838 & $6.6 \pm 2.5$ \\
\hline 1997 & 3 & 17.8 & 07 & & & & & & & & 81 & \\
\hline 1998 & 923 & $13.0=$ & 735 & $5.4=$ & 2.393 & 4.7 & 18 & & 268 & 3.0 & 648 & 1.9 \\
\hline 99 & & & & & & & & & & & & \\
\hline 000 & 5 & & 22 & & 26 & & & & & & 77 & \\
\hline 2001 & 642 & 10. & 068 & 5.2 & 1.064 & $12.8 \pm$ & 20 & & 23 & & 10 & 8.1 \\
\hline 2002 & & 10.3 & 11 & 19.2 & & & & & & & 35 & 5.3 \\
\hline 03 & 0 & & & & & & & & 27 & & 47 & \\
\hline 004 & 1 & & 27 & \pm 7.0 & & & & & 62 & & 41 & \\
\hline 2005 & 062 & $3.8 \pm$ & 0.969 & $0.5 \pm 13.0$ & 1.458 & .3 & & & 1.818 & & 2.609 & $8.2 \pm 2.8$ \\
\hline 2006 & 012 & $6.6 \pm 5.5$ & 0.886 & $9.2 \pm 11.5$ & 1.536 & $4.2 \pm 3.2$ & 1.323 & $5.2 \pm 4.1$ & 2.024 & 3.1 & 2.841 & $2.6 \pm 2.1$ \\
\hline ean & & $0.0 \pm 5.2$ & & $11.3 \pm 4.1$ & & $7.0 \pm 3.0$ & & $5.5 \pm 1.8$ & & 5.2 & & $4.6 \pm 2.3$ \\
\hline
\end{tabular}

\begin{tabular}{|c|c|c|c|c|c|c|c|c|c|c|}
\hline \multirow[t]{2}{*}{ Year } & \multicolumn{2}{|c|}{$\mathrm{Ha}(-2)$} & \multicolumn{2}{|c|}{$\mathrm{Ha}(+2)$} & \multicolumn{2}{|c|}{$\mathrm{Ha}(-1)$} & \multicolumn{2}{|c|}{$\overline{\mathrm{Ha}(+1)}$} & \multicolumn{2}{|c|}{$\mathrm{Ha}(0)$} \\
\hline & Flux & $(e \pm \sigma)$ & Flux & $(\mathrm{e} \pm \sigma)$ & Flux & $(e \pm \sigma)$ & Flux & $(e \pm \sigma)$ & Flux & $(e \pm \sigma)$ \\
\hline 1996 & 36.664 & $9.4 \pm 6.8$ & 29.766 & $11.0 \pm 7.0$ & 45.469 & $9.1 \pm 6.8$ & 42.018 & $11.6 \pm 7.6$ & 48.583 & $11.1 \pm 7.6$ \\
\hline 1997 & 33.714 & $7.0 \pm 8.2$ & 28.349 & $8.1 \pm 7.1$ & 43.930 & $6.8 \pm 7.1$ & 41.554 & $7.9 \pm 9.2$ & 48.475 & $7.4 \pm 8.0$ \\
\hline 1998 & 27.717 & $3.5 \pm 3.2$ & 26.397 & $4.6 \pm 4.0$ & 34.577 & $3.5 \pm 3.8$ & 36.438 & $6.4 \pm 5.5$ & 39.903 & $7.3 \pm 4.9$ \\
\hline 1999 & 29.420 & $1.7 \pm 2.1$ & 27.907 & $1.4 \pm 0.8$ & 37.880 & $1.5 \pm 0.1$ & 42.658 & $3.5 \pm 2.6$ & 48.223 & $2.5 \pm 2.2$ \\
\hline 2000 & 15.153 & $3.9 \pm 4.2$ & 12.928 & $4.4 \pm 3.7$ & 19.099 & $4.6 \pm 3.9$ & 20.783 & $6.6 \pm 5.7$ & 22.577 & $7.5 \pm 6.8$ \\
\hline 2001 & 11.216 & $8.1 \pm 6.3$ & 9.774 & $8.0 \pm 6.2$ & 13.240 & $10.4 \pm 8.4$ & 11.934 & $10.1 \pm 8.4$ & 13.292 & $16.2 \pm 13.0$ \\
\hline 2002 & 12.285 & $4.1 \pm 1.1$ & 12.612 & $6.0 \pm 2.1$ & 18.827 & $4.7 \pm 3.8$ & 16.231 & $14.9 \pm 8.3$ & 19.611 & $12.4 \pm 4.7$ \\
\hline 2003 & 16.800 & $2.5 \pm 2.2$ & 16.071 & $4.6 \pm 4.5$ & 24.724 & $3.6 \pm 2.5$ & 21.440 & $13.5 \pm 9.0$ & 25.761 & $9.8 \pm 4.9$ \\
\hline 2004 & 9.552 & $4.8 \pm 1.5$ & 12.490 & $9.1 \pm 3.2$ & 14.567 & $1.4 \pm 0.4$ & 13.621 & $18.0 \pm 4.9$ & 14.724 & $9.7 \pm 2.5$ \\
\hline 2005 & 7.416 & $4.3 \pm 3.2$ & 10.705 & $9.8 \pm 5.3$ & 9.008 & $8.3 \pm 6.2$ & 9.852 & $32.0 \pm 21.3$ & 8.791 & $26.0 \pm 25.4$ \\
\hline 2006 & 8.489 & $2.6 \pm 2.9$ & 13.613 & $2.6 \pm 2.0$ & 12.402 & $4.2 \pm 1.6$ & 12.362 & $9.3 \pm 4.9$ & 12.101 & $12.8 \pm 5.6$ \\
\hline mean & & $4.7 \pm 2.4$ & & $6.3 \pm 3.1$ & & $5.3 \pm 3.0$ & & $12.2 \pm 7.8$ & & $11.2 \pm 6.1$ \\
\hline
\end{tabular}

\begin{tabular}{ccccccccccc}
\hline Year & \multicolumn{2}{c}{$\mathrm{Hb}(-2)$} & \multicolumn{2}{c}{$\mathrm{Hb}(+2)$} & \multicolumn{2}{c}{$\mathrm{Hb}(-1)$} & \multicolumn{3}{c}{$\mathrm{Hb}(+1)$} & \multicolumn{3}{c}{$\mathrm{Ha}(0)$} \\
& Flux & $(e \pm \sigma)$ & Flux & $(e \pm \sigma)$ & Flux & $(e \pm \sigma)$ & Flux & $(e \pm \sigma)$ & Flux & $(e \pm \sigma)$ \\
\hline 1996 & 10.383 & $7.0 \pm 4.5$ & 8.673 & $6.2 \pm 1.5$ & 11.245 & $6.3 \pm 4.5$ & 11.531 & $6.7 \pm 2.2$ & 12.884 & $7.8 \pm 3.9$ \\
1997 & 9.883 & $3.7 \pm 2.3$ & 8.188 & $3.3 \pm 3.1$ & 11.509 & $4.1 \pm 2.5$ & 10.986 & $4.0 \pm 3.1$ & 13.094 & $4.9 \pm 1.1$ \\
1998 & 7.688 & $2.7 \pm 2.5$ & 7.905 & $2.1 \pm 1.4$ & 8.515 & $2.7 \pm 2.9$ & 9.689 & $1.7 \pm 1.4$ & 10.530 & $1.8 \pm 2.1$ \\
1999 & 6.392 & $4.1 \pm 1.9$ & 6.609 & $5.1 \pm 2.9$ & 6.829 & $6.3 \pm 3.9$ & 8.664 & $4.8 \pm 3.2$ & 9.542 & $6.6 \pm 6.8$ \\
2000 & 2.499 & $2.6 \pm 2.0$ & 2.698 & $1.7 \pm 1.0$ & 3.105 & $3.9 \pm 2.5$ & 3.844 & $3.0 \pm 1.7$ & 4.193 & $5.5 \pm 4.6$ \\
2001 & 2.613 & $4.4 \pm 2.5$ & 2.716 & $5.6 \pm 5.6$ & 3.220 & $4.5 \pm 4.0$ & 3.367 & $2.5 \pm 1.3$ & 3.772 & $8.0 \pm 8.5$ \\
2002 & 2.848 & $5.0 \pm 4.2$ & 3.575 & $4.9 \pm 3.8$ & 4.058 & $5.3 \pm 4.2$ & 4.222 & $5.3 \pm 4.2$ & 4.873 & $6.8 \pm 4.6$ \\
2003 & 4.171 & $4.2 \pm 2.1$ & 4.950 & $3.0 \pm 1.5$ & 6.619 & $2.9 \pm 2.9$ & 6.203 & $3.4 \pm 3.0$ & 7.277 & $4.2 \pm 3.2$ \\
2004 & 2.280 & $5.4 \pm 2.5$ & 3.476 & $3.3 \pm 2.7$ & 3.402 & $4.6 \pm 4.6$ & 3.801 & $3.5 \pm 3.1$ & 4.153 & $3.9 \pm 5.3$ \\
2005 & 2.111 & $4.0 \pm 3.4$ & 3.379 & $5.0 \pm 2.4$ & 2.790 & $4.4 \pm 2.5$ & 3.395 & $7.7 \pm 2.7$ & 3.193 & $9.1 \pm 4.1$ \\
2006 & 2.604 & $2.5 \pm 2.5$ & 3.930 & $1.2 \pm 0.9$ & 3.007 & $2.0 \pm 1.7$ & 4.105 & $2.3 \pm 1.8$ & 3.686 & $3.5 \pm 4.0$ \\
\hline mean & & $4.1 \pm 1.3$ & \multicolumn{10}{c}{$3.8 \pm 1.7$} & \multicolumn{10}{c}{$4.3 \pm 1.4$} & & $4.1 \pm 1.9$ & & $5.6 \pm 2.2$ \\
\hline
\end{tabular}



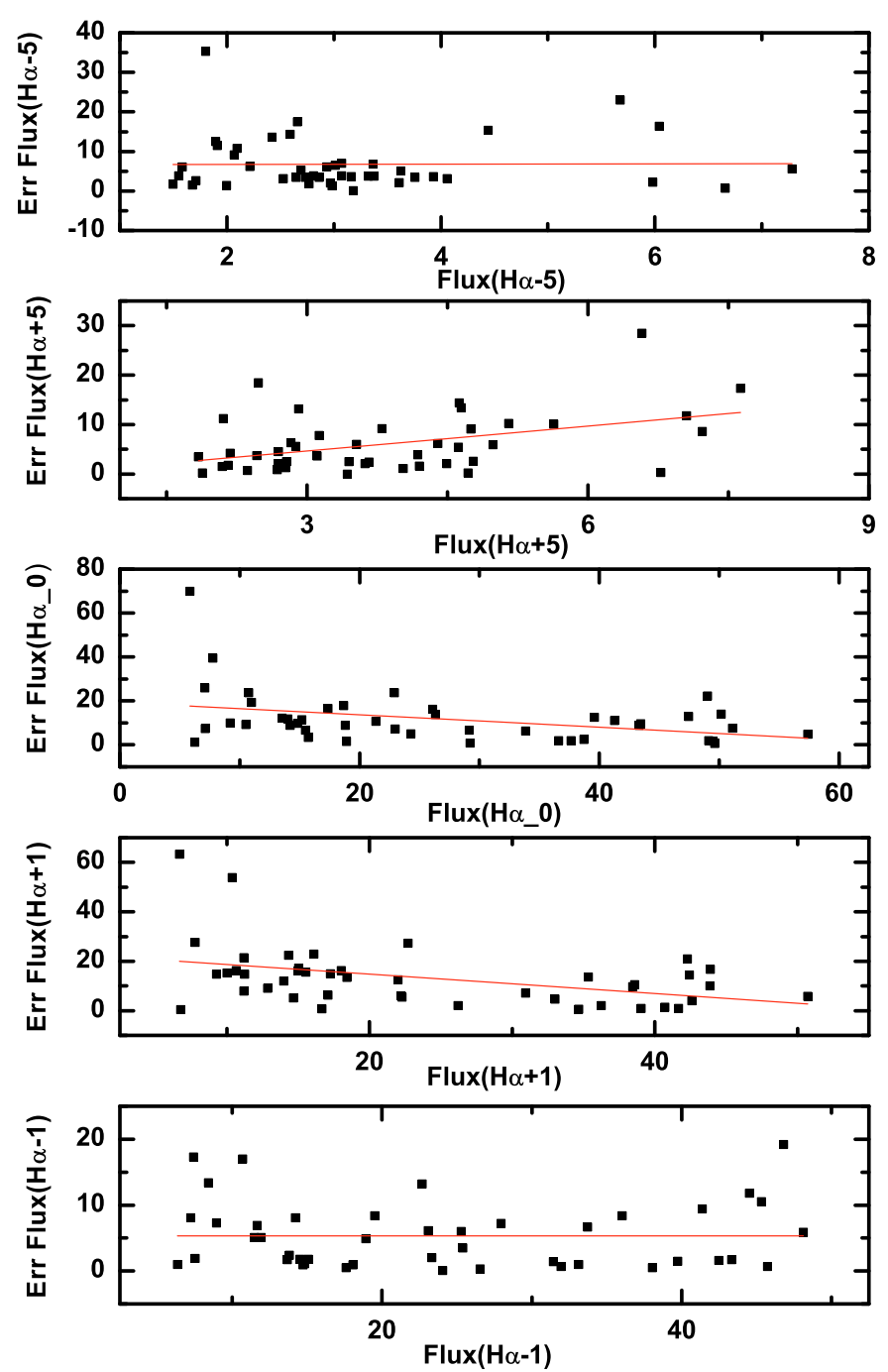

Fig. 9. The flux error measurements (in percent of part line flux) against flux for $-5,-1,0,1,5$ segments of the $\mathrm{H} \alpha$ line. The flux is in units of $10^{-13} \mathrm{erg} \mathrm{cm}^{-2} \mathrm{~s}^{-1}$.

In Fig. 18, we indicate the HeII/HeI versus continuum flux (first panel), BD versus $\mathrm{HeII} / \mathrm{HeI}$ (second panel), and $\mathrm{He} \mathrm{II/H} \beta$ versus continuum flux (third panel). As can be seen from Fig. 18 (first panel), there is a good correlation between the continuum flux and the helium line ratio (the correlation coefficient $r=0.81$ ), which indicates that these lines probably originate in the region photoionized by the continuum source. On the other hand, the Balmer decrement decreases as the ratio of the two helium lines increases (the anti-correlation is a bit weaker here, the correlation coefficient being $r=0.75$ ), indicating that as the ionization becomes stronger, the BD decreases (Fig. 18, second panel).

The photoionization model (Korista \& Goad 2004) illustrates that the Balmer lines should exhibit less flux variation with continuum state than He I, which varies less than He II. In this case, one can expect that $\mathrm{He} \mathrm{II} / \mathrm{H} \beta$ changes far more than $\mathrm{He} \mathrm{II} / \mathrm{He} \mathrm{I}$. The observed changes in the $\mathrm{He} \mathrm{II} / \mathrm{H} \beta$ flux ratio (see Fig. 18, third panel) are probably not caused by differences in the way that these two lines respond to the level of the ionizing continuum flux (see Shapovalova et al. 2008, Fig. 10 in their paper), but rather to changes in the shape of the ionizing SED. In our case, this ratio drops by about 4 times between the high and low
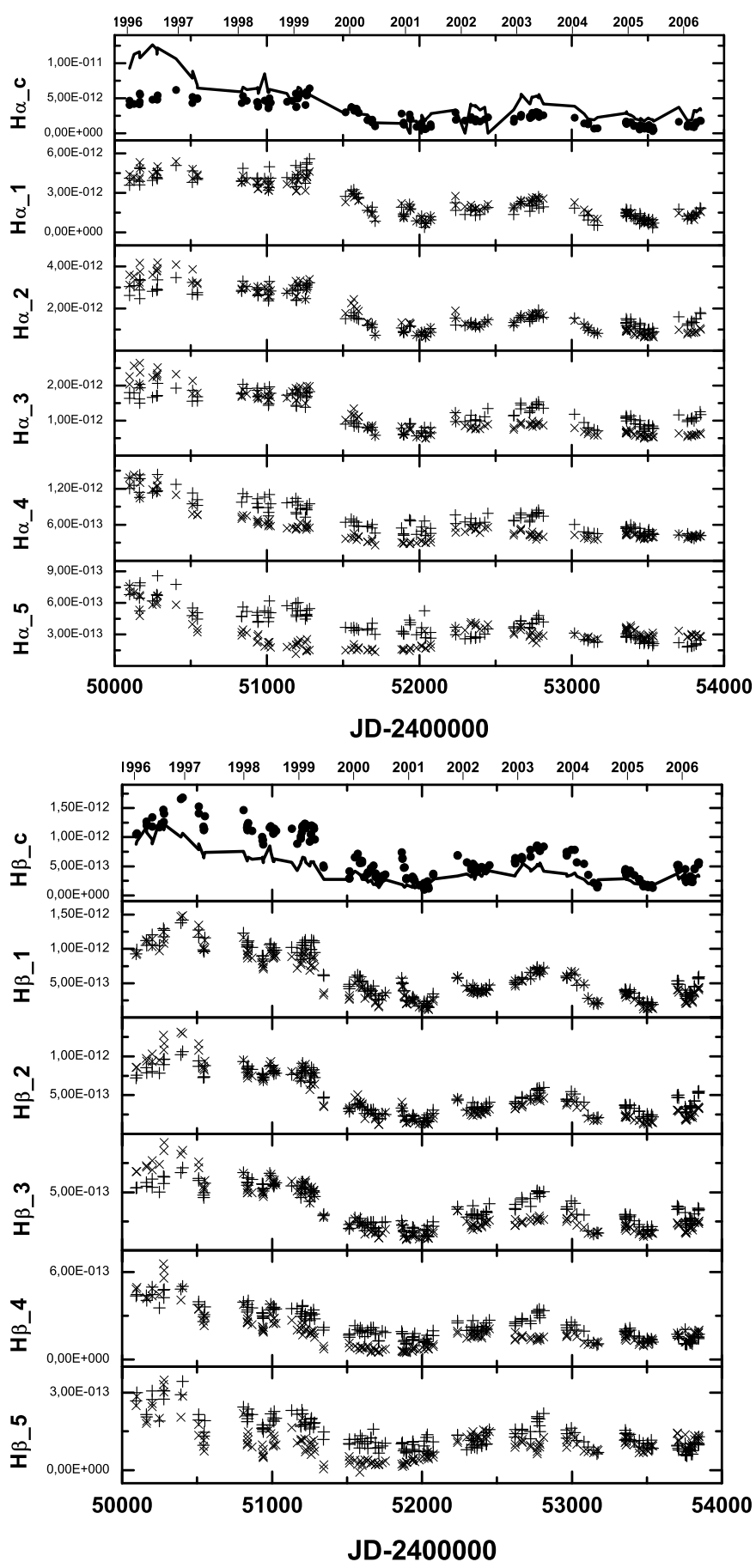

Fig. 10. Light curves of the different $\mathrm{H} \alpha$ (upper panel) and $\mathrm{H} \beta$ (bottom panel) line segments. The segments in the blue wing (numbers from -5 to -1 from Table 3 ) are marked with crosses $(\times)$, and in the red wing (numbers from +5 to +1 from Table 3 ) with plus $(+)$. The abscissae gives the Julian date (bottom) and the corresponding year (top). The ordinate gives the flux in units erg cm $\mathrm{cm}^{-2} \mathrm{~s}^{-1}$. The variation in the continuum flux, which is scaled by different factors (100 and 10 times for $\mathrm{H} \alpha$ and $\mathrm{H} \beta$, respectively) to be comparable with the variation in the central part of the line, is presented with the solid line.

continuum states, while the $\mathrm{He}$ II/He I line ratio changes around 3 times.

There is some connection between the helium and Balmer lines (Fig. 18, left), but the physical properties of the emitting regions of these lines differ probably. One explanation of this correlation may be that part of the Balmer lines originate in the 

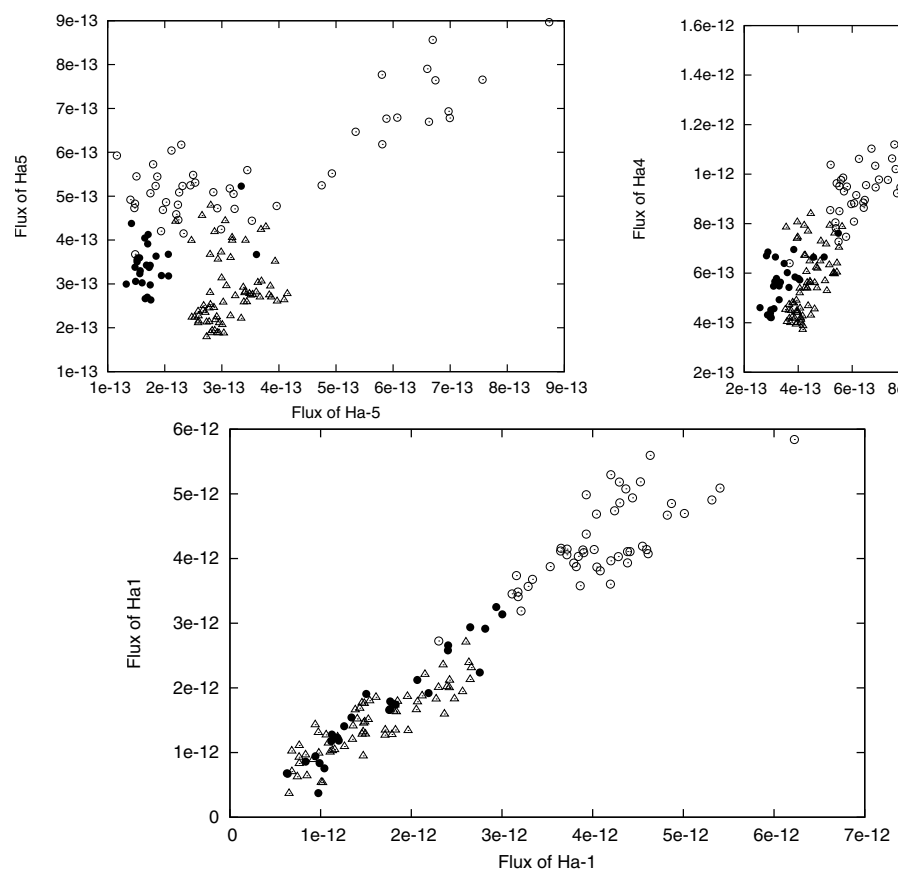
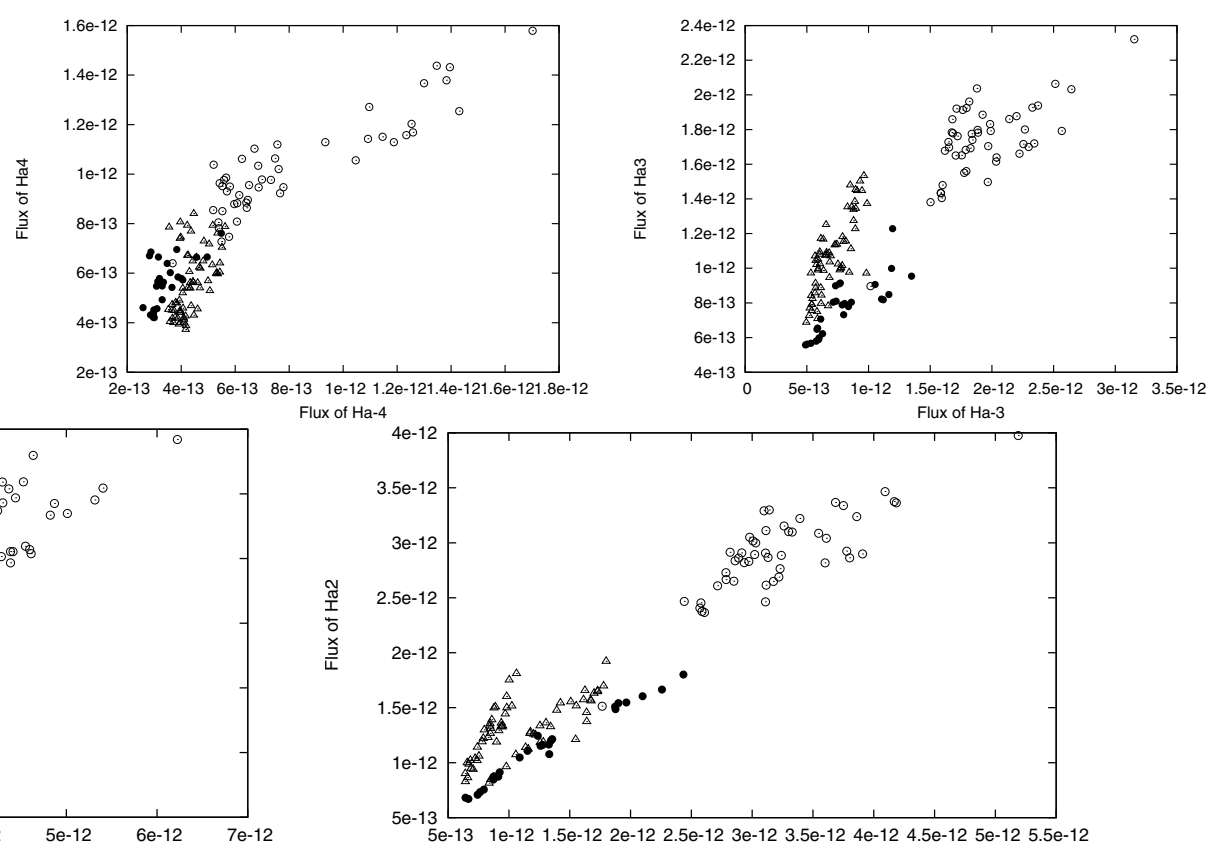

Flux of $\mathrm{Ha}-2$

Fig. 11. The segment to segment response, where the first period (Period I, 1996-1999) is denoted with open circles, the second period (Period II, 2000-2001) with full ones, and the third period (Period III, 2002-2006) with open triangles (Paper I). The flux is given in erg $\mathrm{cm}^{-2} \mathrm{~s}^{-1}$.

same region as He I and He II lines. Since the ratio of He II/ He I is sensitive to changes in temperature and electron density, in the case of a higher He II/ He I ratio, the ionization is higher and the population at a higher level in hydrogen has a higher probability, consequently the ratio of $\mathrm{H} \alpha / \mathrm{H} \beta$ is smaller than in the case of a lower He II/ He I ratio.

\section{Summary of the results}

We have investigated different aspects of broad $\mathrm{H} \alpha$ and $\mathrm{H} \beta$ line variations (shapes, ratios, widths, and asymmetries), so in this section we summarize our results.

\subsection{Summary of the line profile variations}

We summarize here the main results of the line profile variations in NGC 4151:

i) the line profile of $\mathrm{H} \alpha$ and $\mathrm{H} \beta$ varied during the monitoring period, exhibiting both blue (1996-1999) and red (2002-2006) asymmetry. We observe bumps (shelves or an additional emission) in the blue region in 1996-1997 at $V_{r} \sim-4000$ and $-2000 \mathrm{~km} \mathrm{~s}^{-1}$, in 1998-99 at $V_{r} \sim-2000 \mathrm{~km} \mathrm{~s}^{-1}$, and in 2000-2001 at -2000 and $-500 \mathrm{~km} \mathrm{~s}^{-1}$ already. However, in 2002-2004 these details disappeared in the blue wing of both lines. It became steeper than the red one and did not contain any noticeable features. In 2002, a distinct bump appeared in the red wing of both lines at $+3100 \mathrm{~km} \mathrm{~s}^{-1}$. The radial velocity of a bump in the red wing changed from $+3100 \mathrm{~km} \mathrm{~s}^{-1}$ in 2002 to $2100 \mathrm{~km} \mathrm{~s}^{-1}$ in 2006 . The appearance of the blue and red bumps was possibly related to a jet component;

ii) in 2005 (May-June), when the nucleus of NGC 4151 experienced its minimum activity state, the line profiles had a double-peaked structure with two distinct peaks (bumps) at radial velocities of $-2586,+2027 \mathrm{~km} \mathrm{~s}^{-1}$ in $\mathrm{H} \beta$ and -1306 , $+2339 \mathrm{~km} \mathrm{~s}^{-1}$ in $\mathrm{H} \alpha$;
Table 5. Yearly-averaged and period-averaged variations in the integral Balmer decrement (BD) and continuum fluxes.

\begin{tabular}{lllll}
\hline \hline Year & \multicolumn{2}{l}{$F(\mathrm{H} \alpha) / F(\mathrm{H} \beta) \pm \sigma$} & \multicolumn{2}{l}{$F(5100) \pm \sigma^{a}$} \\
\hline 1996 & 3.65 & 0.51 & 11.18 & 1.55 \\
1997 & 3.39 & 0.36 & 7.62 & 1.03 \\
1998 & 3.58 & 0.35 & 6.08 & 1.19 \\
1999 & 4.49 & 0.74 & 5.12 & 1.62 \\
2000 & 5.01 & 0.62 & 2.75 & 1.33 \\
2001 & 5.43 & 1.07 & 1.87 & 0.47 \\
2002 & 3.67 & 0.44 & 3.59 & 0.29 \\
2003 & 3.16 & 0.27 & 4.84 & 0.58 \\
2004 & 3.70 & 0.29 & 2.44 & 0.57 \\
2005 & 3.96 & 0.54 & 1.99 & 0.18 \\
2006 & 3.58 & 0.36 & 2.60 & 0.80 \\
\hline mean (1996-1998): & 3.54 & 0.13 & 8.29 & 2.62 \\
\hline mean 1999(Jan.-Apr.): & 4.21 & 0.59 & 5.89 & 0.56 \\
1999 (Dec.): & 5.33 & & 2.78 & 0.12 \\
\hline mean (2000-2001): & 5.22 & 0.30 & 2.31 & 0.62 \\
\hline mean (2002-2006): & 3.61 & 0.29 & 3.09 & 1.14 \\
\hline
\end{tabular}

${ }^{a}$ In units of $10^{-14} \mathrm{erg} \mathrm{cm}^{-2} \mathrm{~s}^{-1} \AA^{-1}$.

Columns: 1 - year or yearly-intervals; $2-F(\mathrm{H} \alpha) / F(\mathrm{H} \beta), \mathrm{H} \alpha$ and $\mathrm{H} \beta$ flux ratio or integrated Balmer Decrement (BD); $3-\sigma(F(\mathrm{H} \alpha) / F(\mathrm{H} \beta))$, the estimated $F(\mathrm{H} \alpha) / F(\mathrm{H} \beta)$ error; $4-F(5100)$, continuum flux at $\lambda 5100 \AA ; 5-\sigma$, estimated continuum flux error. The last four lines provide the mean $\mathrm{BD}$, mean continuum flux, and their estimated errors in year-periods.

iii) in 1996-2001, we observed a broad deep absorption line in $\mathrm{H} \beta$ at a radial velocity that took values from $V_{r} \sim-1000$ to $V_{r} \sim-400 \mathrm{~km} \mathrm{~s}^{-1}$;

iv) the $F W H M$ is not correlated with the continuum flux, while the asymmetry tends to anti-correlate with it (coefficient correlation $r \sim-0.5$ ). The FWHM and asymmetry in $\mathrm{H} \beta$ are greater than in $\mathrm{H} \alpha$, which may be caused by $\mathrm{H} \beta$ originating closer to the $\mathrm{SMBH}$, thus explaining its larger widths; 
A. I. Shapovalova et al.: Variability of NGC 4151. II.
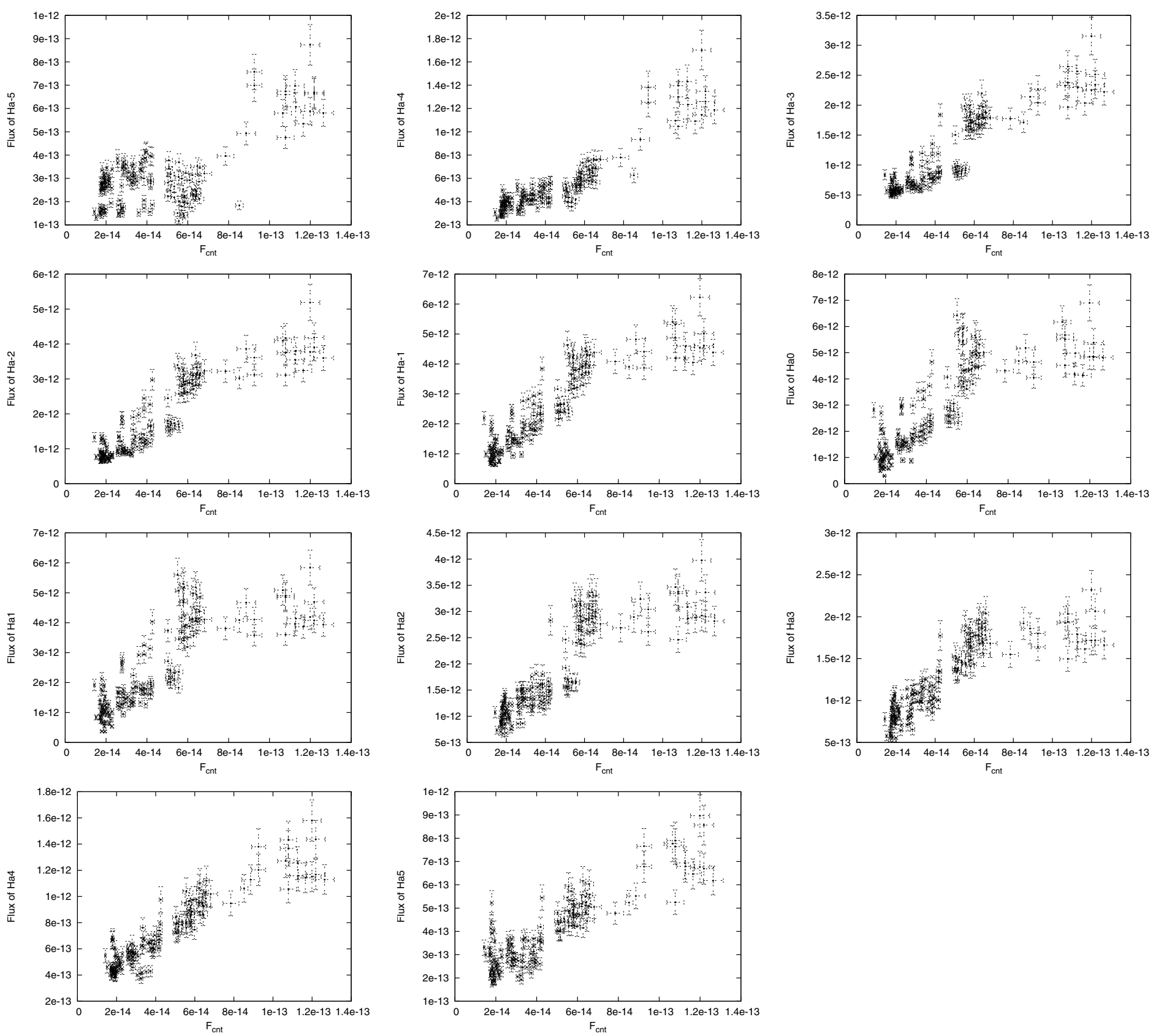

Fig. 12. The flux of different segments of the line as a function of the continuum flux. The line flux is given in erg $\mathrm{cm}^{-2} \mathrm{~s}^{-1}$ and the continuum flux in $\operatorname{erg} \mathrm{cm}^{-2} \mathrm{~s}^{-1} \AA^{-1}$.

v) we divided the line profiles into 11 identical segments, each of width $1000 \mathrm{~km} \mathrm{~s}^{-1}$ and investigated the correlations between segments and continuum flux and between segments and segments flux. We found that the far red wings (from 3500 to $5500 \mathrm{~km} \mathrm{~s}^{-1}$ ) and central (at $V_{r}=$ $\pm 3500 \mathrm{~km} \mathrm{~s}^{-1}$ ) segments for the continuum flux $F_{\mathrm{c}}<$ $7 \times 10^{-14} \mathrm{erg} \mathrm{cm}^{-2} \mathrm{~s}^{-1} \mathrm{~A}^{-1}$ respond to the continuum almost linearly, and that these segments probably originate in the part of the BLR ionized by the AGN source;

vi) the central $\left( \pm 3500 \mathrm{~km} \mathrm{~s}^{-1}\right)$ segments for $F_{\mathrm{c}}>7 \times$ $10^{-14} \mathrm{erg} \mathrm{cm}^{-2} \mathrm{~s}^{-1} \mathrm{~A}^{-1}$ do not show any linear relation with continuum flux. In periods of high activity, these line segments probably partly originate in substructures that are not photoioninized by the AGN continuum; vii) the far blue wing (from -5500 to -4500 ) seems to originate in a separate region, so it does not respond to the continuum flux variation as well as to the variation of the other line segments, except in the case of high line flux, where it responds to the far red wing (see Fig. 12). This may indicate that the far blue wing is emitted by a distinct region that is not photoionized, and also that the emission is highly blueshifted (as e.g., an outflow with velocities $>3500 \mathrm{~km} \mathrm{~s}^{-1}$ );

viii) the far red wing is very sensitive to the continuum flux variation, and thus probably originates in a region closest to the center, i.e., this part of the line seems to be purely photoionized by the AGN source. 

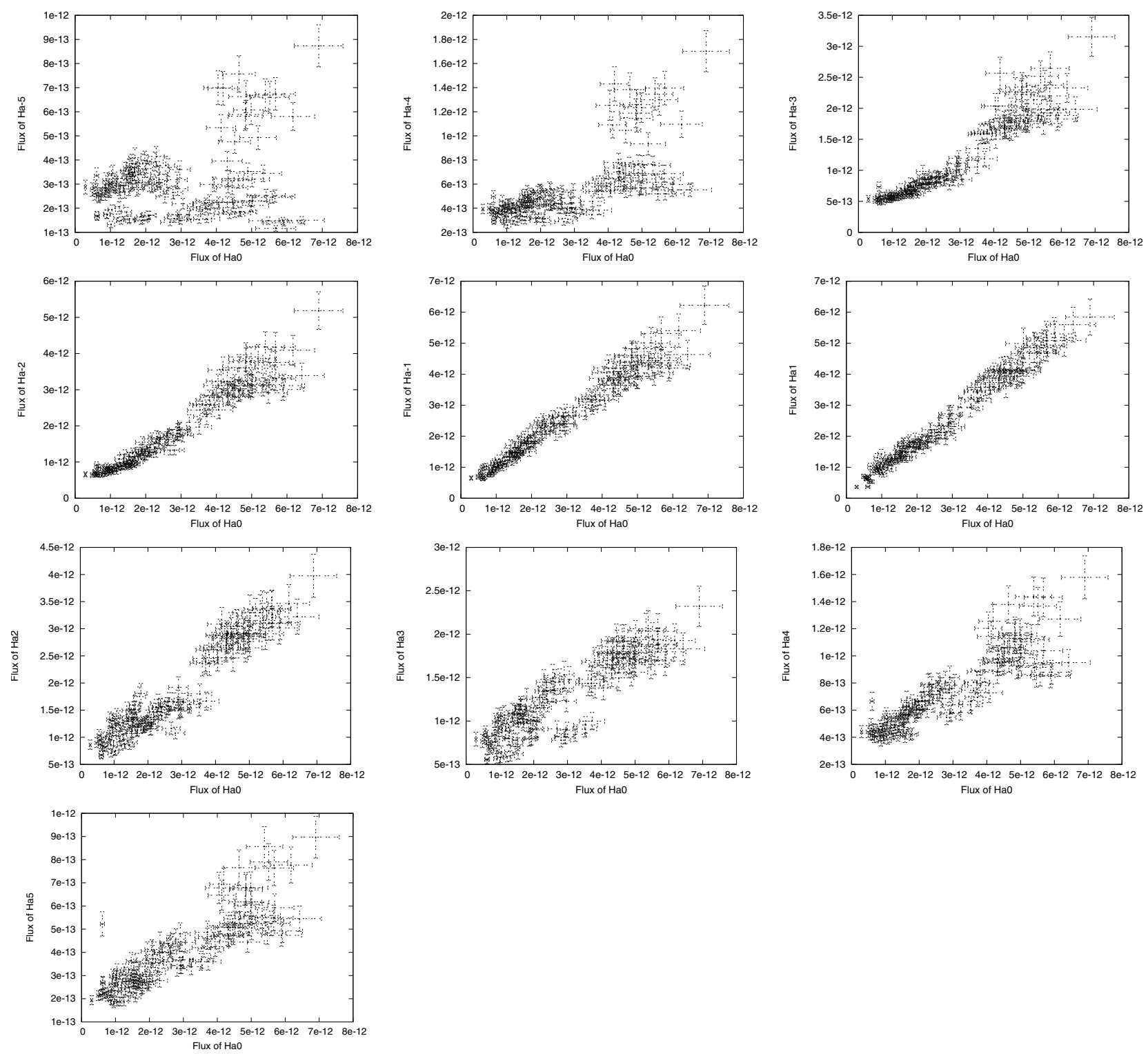

Fig. 13. The flux of different $\mathrm{H} \alpha$ segments as a function of the flux of the central $\mathrm{H} \alpha$ segment. The line flux is given in erg $\mathrm{cm}^{-2} \mathrm{~s}^{-1}$.

From all above mentioned, one can conclude that the broad lines of NGC 4151 are produced by least three kinematically (and physically) distinct regions.

\subsection{Summary of the $B D$ variations}

During the monitoring period, the Balmer decrement $(\mathrm{BD}=$ $F(\mathrm{H} \alpha) / F(\mathrm{H} \beta))$ varied from 2 to 8 . It is also interesting that BDs differ along line profiles. In 1996-1998, there was no significant correlation between the BD and the continuum flux (the continuum flux was $\left.F_{\mathrm{c}} \sim(6-12) \times 10^{-14} \mathrm{erg} \mathrm{cm}^{-2} \mathrm{~s}^{-1} \mathrm{~A}^{-1}\right)$. In 1999-2001, maximal variations in the BD were observed, especially in the blue part of lines. The maximal value of the BD along the line profiles strongly differed: in 1996-2001, the BD reached its maximum at $V_{r} \sim \pm 1000 \mathrm{~km} \mathrm{~s}^{-1}$ and in 2002-2006 the BD had 2 peaks - at velocities from -2000 to -1000 and at $V_{r} \sim 3000 \mathrm{~km} \mathrm{~s}^{-1}$ for somewhat smaller (by $\sim(0.5-1)$ ) values of the BD. In the last case, it is possible that the second bump (the fainter one) is caused by the interaction between the receding sub-parsec jet and environment.

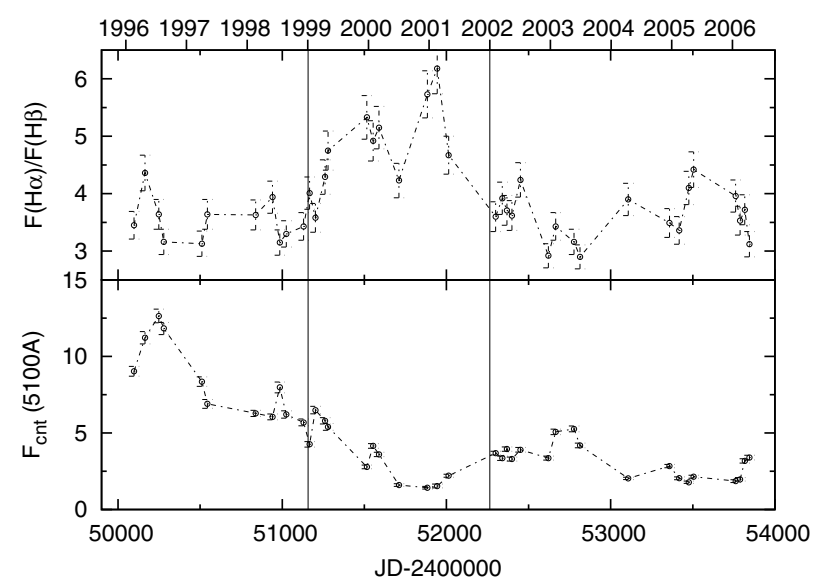

Fig. 14. Variations in the integrated Balmer decrement $\mathrm{BD}=$ $F(\mathrm{H} \alpha) / F(\mathrm{H} \beta$ (upper panel) and the continuum flux at $\lambda 5100 \AA$ (bottom panel) in 1996-2006. The abscissae gives the Julian date (bottom) and the corresponding year (top). The continuum flux is in units $10^{-14} \mathrm{erg} \mathrm{cm}^{-2} \mathrm{~s}^{-1} \AA^{-1}$. The vertical lines correspond to years 1999 and 2002 . 


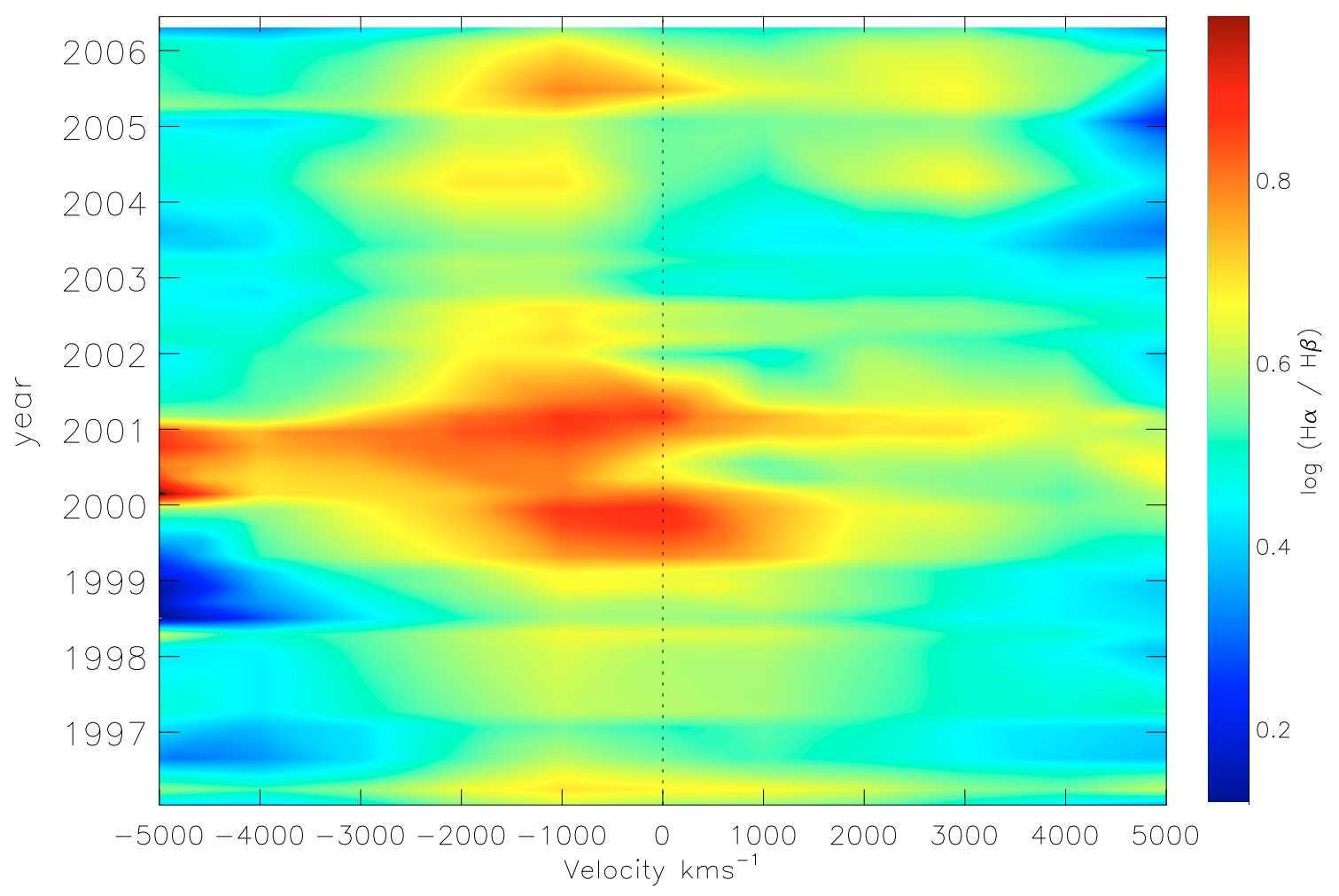

Fig. 15. The variations in the Balmer decrement for different parts of the broad emission lines. The flux is given in $\operatorname{erg~cm}^{-2} \mathrm{~s}^{-1} \AA^{-1}$.

The different values of the $\mathrm{BD}$ observed during the monitoring period (as well as different values of the BD along the profiles) are also indicative of a multicomponent origin of the broad lines. These different ratios may be caused by absorption, but also by different physical conditions in different parts of the BLR.

\section{Discussion}

It is interesting to compare our results with those found in the UV and X rays. Crenshaw \& Kraemer (2007) found a width of $1170 \mathrm{~km} \mathrm{~s}^{-1}(F W H M)$ for the UV emission lines significantly smaller than our results for the Balmer lines ( $F W H M \sim 5000-7000 \mathrm{~km} \mathrm{~s}^{-1}$, around 5-6 times smaller). This can be interpreted as the existence of an intermediate component between the broad and narrow (emission) line regions (see e.g., Popović et al. 2009). In the same paper, they found evidence that the UV emission lines originate in the same gas responsible for most of the UV and X-ray absorption. The absorption can be seen in outflow at a distance of $0.1 \mathrm{pc}$ from the central nucleus. It is also interesting that Crenshaw \& Kraemer (2007) favor a magnetocentrifugal acceleration (e.g., in an accretion disk wind) over those that rely on radiation or thermal expansion as the principal driving mechanism of the mass outflow. Obscuration should play an important role, but we found that while absorption can clearly be detected in the $\mathrm{H} \beta$ line, the $\mathrm{H} \alpha$ line displays a small amount of absorption (see Figs. 2 and 3). Absorption in the $\mathrm{H} \beta$ line was previously reported by Hutchings et al. (2002), therefore obscuration of the optical continuum should be present in NGC 4151. The location of the obscuring material was estimated by Puccetti et al. (2007). They analyzed the X-ray variability of the nucleus. They found that the location of the obscuring matter is within $3.4 \times 10^{4}$ Schwarzschild radii of the central $\mathrm{X}$-ray source and suggested that absorption

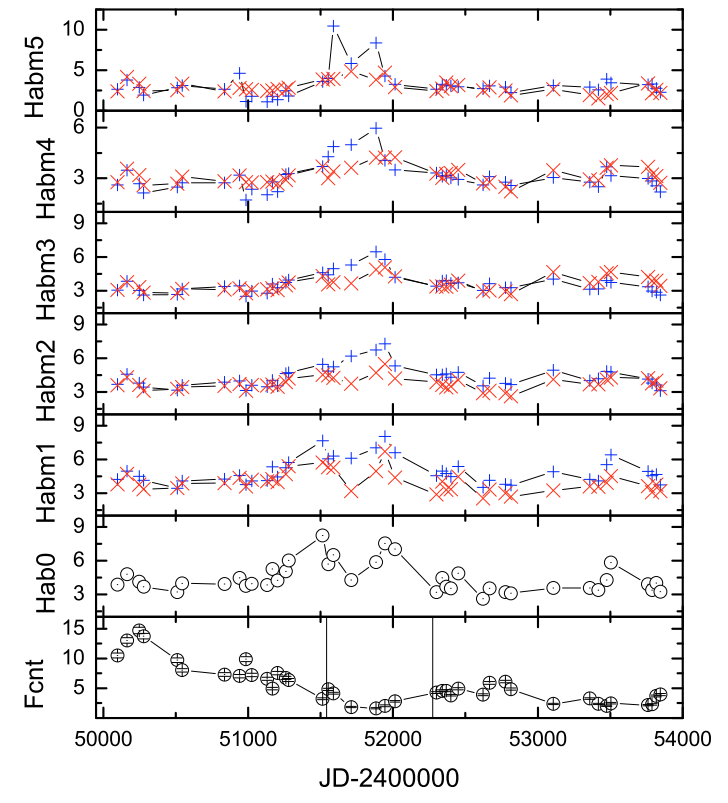

Fig. 16. Variations in the BD of different segments of the line profiles and in the continuum flux (bottom panel) in 1996-2006. The BD of segments in the blue wing (numbers from -5 to -1 from Table 3 ) are denoted with plus $(+)$, in red wing (numbers from +5 to +1 from Table 3 ) with crosses $(\times)$, and of central part (number 0 ) with open circles. The vertical lines correspond to years 1999 and 2002. The abscissae shows the Julian date (bottom) and the corresponding year (top). The ordinate shows the BD for different segments of the line profile. The continuum flux is in units $10^{-14} \mathrm{erg} \mathrm{cm}^{-2} \mathrm{~s}^{-1} \AA^{-1}$.

variability plays a crucial role in the observed flux variability of NGC 4151.

We discuss some possible formation scenarios for the BLR. As we mentioned above, the outflow is probably induced by 
A\&A 509, A106 (2010)
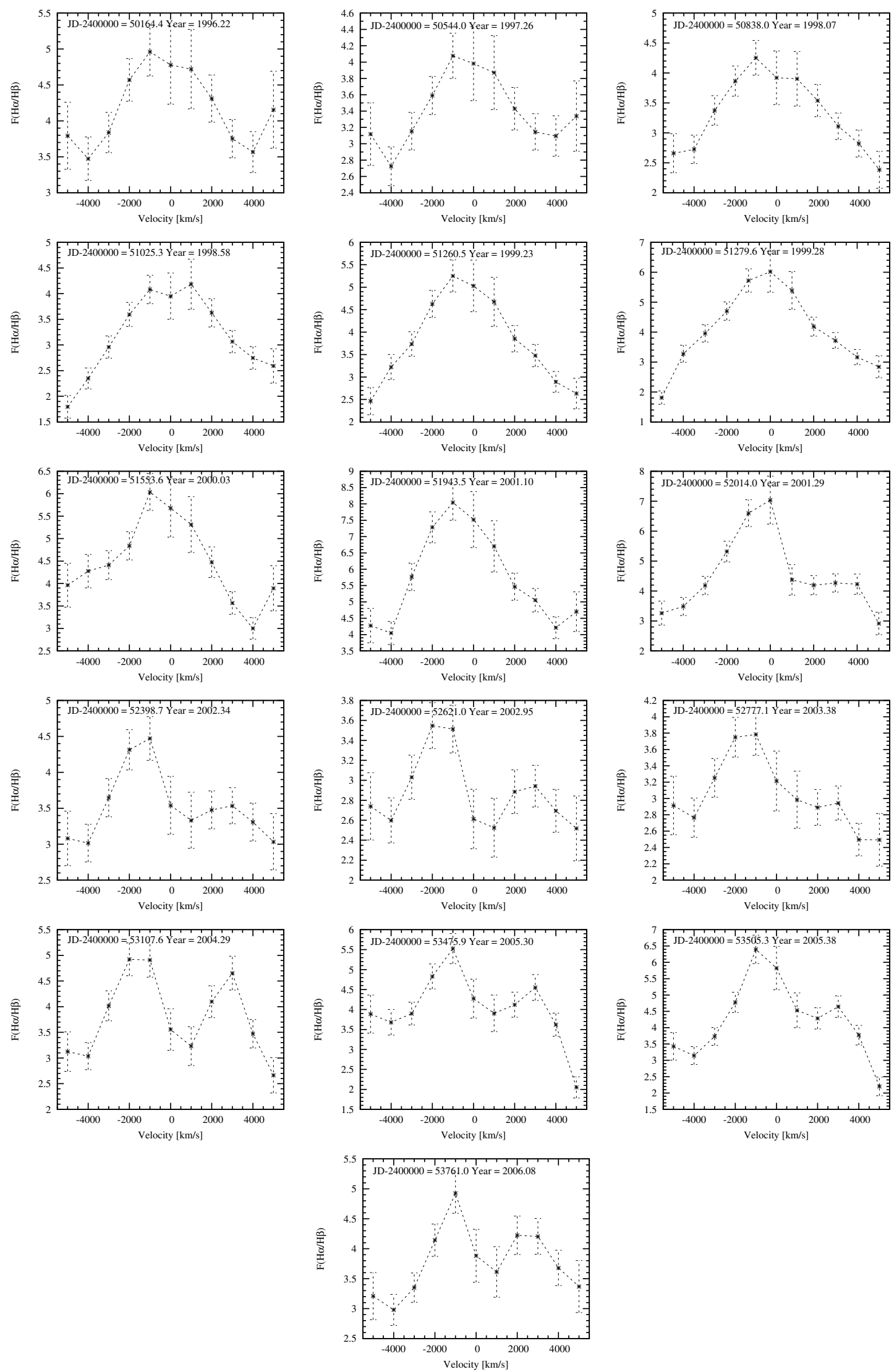

Fig. 17. Variations in the Balmer decrement $\mathrm{BD}=F(\mathrm{H} \alpha) / F(\mathrm{H} \beta)$ as a function of the radial velocity for monthly-averaged spectra each year of the monitoring period. The abscissae indicates the radial velocities relative to the narrow components. The monthly-averaged Julian date and the corresponding year are given at the top of each plot. 
A. I. Shapovalova et al.: Variability of NGC 4151. II.
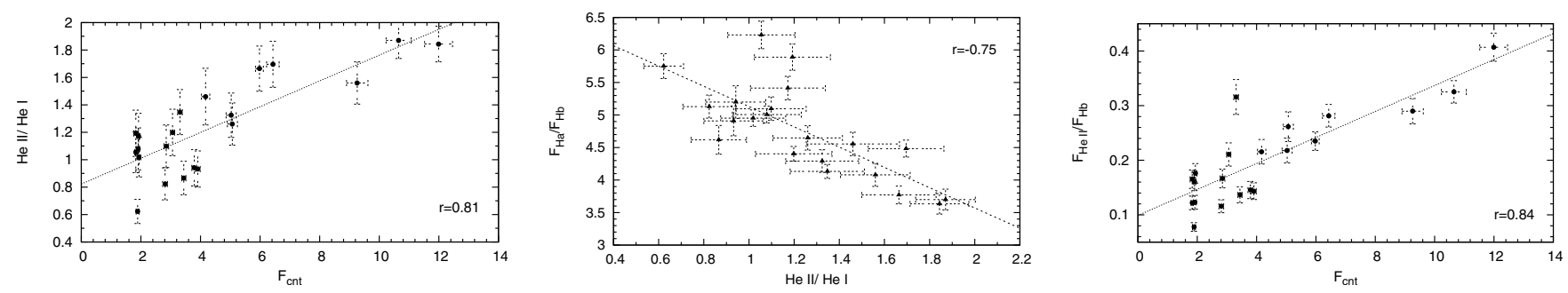

Fig. 18. Left: the variation of the ratio of the helium He II $\lambda 4686$ and He I $\lambda 5876$ lines as a function of the continuum flux. Middle: the BD as a function of the helium line ratio. Right: the variation of the ratio of the helium He II $\lambda 4686$ and $\mathrm{H} \beta$ lines as a function of the continuum flux. The continuum flux is in units $10^{-14} \mathrm{erg} \mathrm{cm}^{-2} \mathrm{~s}^{-1} \AA^{-1}$.

the magnetocentrifugal acceleration, and starts very close to the black hole. If we adopt a black hole mass of around $4 \times 10^{7} M_{\odot}$ (obtained from stellar dynamical measurements, see Onken et al. 2008, which is in agreement with Bentz et al. 2006), the acceleration (and line emission) starts at $\sim 10^{-4}$ pc from the black hole and the outflow emits until reaching a distance of $\sim 0.01 \mathrm{pc}$, taking into account the absorption velocities (e.g. Hutchings et al. 2002) of around $-1000 \mathrm{~km} \mathrm{~s}^{-1}$,

As mentioned above, the broad $\mathrm{H} \alpha$ and $\mathrm{H} \beta$ lines show different widths and asymmetries during the monitoring period, indicating a complex structure of the BLR. We also found that the line profiles of $\mathrm{H} \alpha$ and $\mathrm{H} \beta$ could be different at the same epoch. Therefore, one should consider a multi-component origin of these lines.

To propose a BLR model, one should take into account that: a) there is an absorption component in the blue part of lines that indicate that some kind of outflow may start in the BLR (see Sect. 3.2). Crenshaw \& Kraemer (2007) also reported a mass outflow from the nucleus of NGC 4151, but they confirmed the observed outflow in the intermediate line region (ILR); b) the flux in the far red wing correlates well with the continuum flux, indicating that it originates in an emission region very close to the continuum source, i.e., to the central black hole; c) the flux of the far blue wing does not correlate with the continuum, which may be indicative of some kind of shock contribution to the Balmer line.

All of these results indicate that an outflow probably exists in the BLR. In this case, complex line profiles (with different features) can be expected because of changes in the outflowing structure, as is often seen in the narrow lines observed in the jetinduced shocks (see e.g., Whittle \& Wilson 2004). Of course, we cannot exclude there being some contribution of different regions to the composite line profile, as e.g., there can be also contribution of the ILR which may be with an outflow geometry. In a forthcoming paper, we propose to compare the observational results with those predicted by various models of the kinematics and structure of the BLR (e.g., a bi-conical outflow, an accelerating outflow (wind), a Keplerian disk, jets, etc.).

We note that the line profiles change during the monitoring period, especially after 2002, when the "red bump" appears. After that, the asymmetry of both lines (see Table 2) and BD exhibit different behaviors than in the period 1996-2002. In the III period, the line profiles of $\mathrm{H} \alpha$ and $\mathrm{H} \beta$ very much changed in shape from double-peaked profiles (see Figs. 1 and 2) to quite asymmetrical profiles (as observed in 2006).

The integrated Balmer decrement reached a maximum in 1999-2001 (see Fig. 14). The BD changes shape from 2002, showing two peaks in its BD versus velocity field profile. This is probably connected to strong inhomogeneities in the distribution of the absorbing material during different periods of monitoring. Since the line flux in $\mathrm{H} \alpha$ and $\mathrm{H} \beta$ at low fluxes $\left(<7 \times 10^{-14} \mathrm{erg} \mathrm{cm}^{-2} \mathrm{~s}^{-1} \mathrm{~A}^{-1}\right)$ correlates well with that of the continuum (Paper 1), we infer that the change in the integrated Balmer decrement during 1999-2001 is also caused, at least partially, by changes in the continuum flux. Indeed, when the ionization parameter decreases for a constant density plasma, an increase in $F(\mathrm{H} \alpha) / F(\mathrm{H} \beta)$ intensity ratio is expected (see for instance Wills et al. 1985). This is because of the decrease in the excitation state of the ionized gas, since when the temperature of the ionized zone gets smaller, the population of the upper levels with respect to the lower decreases. In 1996-1998, BDs did not correlate with continuum, i.e., the main cause of BD variations is not related to the active nucleus and the shock initiated emission is probably dominant. We detected that the FWHM of lines does not correlate with the continuum. This confirms our assumption that broad lines can form in several (three) different subsystems or that the emission is affected by an outflow that produces shock-initiated emission.

\section{Conclusions}

This work is intended to proceed Paper I (Shapovalova et al. 2008) and has entailed a detailed analysis of the broad $\mathrm{H} \alpha$ and $\mathrm{H} \beta$ line profile variations during the 11-year period of monitoring (1996-2006). From this study (Sect. 3), it follows that the BLR in NGC 4151 is complex, and that broad emission lines represent the sums of components formed in different subsystems:

1. The first component is photoionized by the AGN continuum (far red line wings, $V_{r} \sim+4000$ and $+5000 \mathrm{~km} \mathrm{~s}^{-1}$ and central (at $V_{r}= \pm 3500 \mathrm{~km} \mathrm{~s}^{-1}$ ) segments for continuum flux $\left.F_{\mathrm{c}}<7 \times 10^{-14} \mathrm{erg} \mathrm{cm}^{-2} \mathrm{~s}^{-1} \mathrm{~A}^{-1}\right)$. This region is the closest to the SMBH.

2. The second component varies independently of changes in the AGN continuum (far blue line wings, $V_{r} \sim$ $-5000 \mathrm{~km} \mathrm{~s}^{-1} ;-4000 \mathrm{~km} \mathrm{~s}^{-1}$ ). It is possibly generated by shocks initiated by an outflow.

3. The third component, where the central parts of lines $\left(V_{r}<4000 \mathrm{~km} \mathrm{~s}^{-1}\right)$ are formed, in high fluxes $F_{\mathrm{c}}>7 \times$ $10^{-14} \mathrm{erg} \mathrm{cm}^{-2} \mathrm{~s}^{-1} \mathrm{~A}^{-1}$ is also independent of the AGN continuum (possibly, outflow and jet).

Finally, we can conclude that the BLR of NGC 4151 may not be purely photoionized, i.e., besides photoionization, there could be some internal shocks contributing to the broad lines (see also discussion in Paper I). There are at least three different subregions with different velocity fields and probably different physical conditions, which produce complex variability in the broad lines of NGC 4151 and changes in the line profile (which are very often temporarily bumps). Our investigations indicate that reverberation mapping might not be valid as a tool to determine the BLR size in this AGN and that this AGN is not perfect for this method. 
Consequently, the results for the BLR size of NGC 4151 (given in the introduction) should be interpreted with caution.

Acknowledgements. Authors would like to thank Suzy Collin for her suggestions how to improve this paper. Also, we thank the anonymous referee for very useful comments. This work was supported by INTAS (grant N960328), RFBR (grants N97-02-17625 N00-02-16272, N03-02-17123 and 0602-16843, 09-02-01136), State program "Astronomy" (Russia), CONACYT research grant 39560-F and 54480 (México) and the Ministry of Science and Technological Development of Republic of Serbia through the project Astrophysical Spectroscopy of Extragalactic Objects (146002).

\section{References}

Abrassart, A., \& Czerny, B. 2000, A\&A, 356, 475

Anderson, K. S. 1974, ApJ, 189, 195

Anderson, K. S., \& Kraft, R. P. 1969, ApJ, 158, 859

Bentz, M. C., Denney, K. D., Cackett, E. M., et al. 2006, ApJ, 651, 775

Boller, Th., Brandt, W. N., \& Fabian, A. C. 1997, MNRAS, 289, 393

Brandt, W. N., Boller, Th., Fabian, A. C., \& Ruszkowski, M. 1999, MNRAS, 303, L53

Clavel, J., Boksenberg, A., Bromage, G. E., et al. 1990, MNRAS, 246, 668

Collin-Souffrin, S., Czerny, B., Dumont, A.-M., \& Zycki, P. T. 1996, A\&A, 314, 393

Crenshaw, D. M., \& Kraemer, S. B. 2007, ApJ, AJ, 659, 250

Crenshaw, D. M., Kraemer, S. B., Hutchings, J. B., et al. 2000, AJ, 120, 1731

Czerny, B., Doroshenko, V. T., Nikolajuk, M., et al. 2003, MNRAS, 342, 1222

Griem, H. R. 1997, Principles of plasma spectroscopy (Cambridge: Cambridge University Press)

Goad, M. R., Korista, K. T., \& Knigge, C. 2004, MNRAS, 352, 277

Hutchings, J. B., Crenshaw, D. M., Danks, A. C., et al. 1999, AJ, 118, 2101

Hutchings, J. B., Crenshaw, D. M., Kraemer, S. B., et al. 2002, AJ, 124, 2543
Kollatschny, W., \& Dietrich, M. 1997, A\&A, 323, 5

Korista, K. T., \& Goad, M. R. 2004, ApJ, 606, 749

Lyuty, V. M. 2005, Pis'ma in Astron. Zh. 31, 723 (rus)

Maoz, D., Netzer, H., Mazeh, T., et al. 1991, ApJ, 367, 493

Newman, J. A., Eracleous, M., Filippenko, A. V., \& Halpern, J. P. 1997, ApJ, 485,570

Onken, C. A., Valluri, M., Peterson, B. M., et al. 2007, ApJ, 670, 105

Peterson, B. M., \& Cota, S. A. 1988, ApJ, 330, 111

Popović, L.Č., Mediavilla, E., Bon, E., \& Ilić, D. 2004, A\&A, 423, 909

Popović, L.Č., Smirnova, A. A., Kovačević, J., Moiseev, A. V., \& Afanasiev, V. L. 2009, AJ, 137, 3548

Puccetti, S., Fiore, F., Risaliti, G., et al. 2007, MNRAS, 377, 607

Risaliti, G., Elvis, M., \& Nicastro, F. 2002, ApJ, 571, 234

Schurch, N. J., \& Warwick, R. S. 2002, MNRAS, 334, 811

Sergeev, S. G., Pronik, V. I., Sergeeva, E. A., \& Malkov, Yu. F. 1999, ApJS, 121, 159

Sergeev, S. G., Pronik, V. I., \& Sergeeva, E. A. 2001, ApJ, 554, 245

Shapovalova, A. I., Doroshenko, V. T., Bochkarev, N. G., et al. 2004, A\&A, 422, 925

Shields, J. C., Ferland, G. J., \& Peterson, B. M. 1995, ApJ, 441, 507

Sulentic, J. W., Marziani, P., \& Dultzin-Hacyan, D. 2000, ARA\&A, 38, 521

Shapovalova, A. I., Popović, L. Č, Collin, S., et al. 2008, A\&A, 486, 99

Ulrich, M.-H. 2000, A\&ARv, 10, 135

Ulrich, M.-H., \& Horne, K. 1996, MNRAS, 283, 748

Ulvestad, J. S., Wong, D. S., Taylor, G. B., Gallimore, J. F., \& Mundell, C. G. 2005, AJ, 130, 936

Vlasyak, V. V. 1993, Bull. Spec. Astrophys. Obs., 36, 107

Wanders, I., \& Peterson, B. M. 1996, ApJ, 466, 174

Waymann, R. J., Morris, S. L., Gray, M. E., \& Hutchings, J. B. 1997, ApJ, 483, 717

Whittle, M., \& Wilson, A. 2004, AJ, 127, 606

Wills, B. J., Netzer, H., \& Wills, D. 1985, ApJ, 288, 94 